\title{
ON THE NODAL SETS OF TORAL EIGENFUNCTIONS
}

\author{
JEAN BOURGAIN AND ZEÉV RUDNICK
}

\begin{abstract}
We study the nodal sets of eigenfunctions of the Laplacian on the standard $d$-dimensional flat torus. The question we address is: Can a fixed hypersurface lie on the nodal sets of eigenfunctions with arbitrarily large eigenvalue? In dimension two, we show that this happens only for segments of closed geodesics. In higher dimensions, certain cylindrical sets do lie on nodal sets corresponding to arbitrarily large eigenvalues. Our main result is that this cannot happen for hypersurfaces with nonzero Gauss-Kronecker curvature.

In dimension two, the result follows from a uniform lower bound for the $L^{2}$-norm of the restriction of eigenfunctions to the curve, proved in an earlier paper [1. In high dimensions we currently do not have this bound. Instead, we make use of the real-analytic nature of the flat torus to study variations on this bound for restrictions of eigenfunctions to suitable submanifolds in the complex domain. In all of our results, we need an arithmetic ingredient concerning the cluster structure of lattice points on the sphere. We also present an independent proof for the two-dimensional case relying on the "abc-theorem" in function fields.
\end{abstract}

\section{Contents}

1. Introduction and statement of results

1.1. Dimension $d=2$

1.2. Higher dimensions

1.3. About the proofs

1.4. The sphere and Legendre polynomials

2. Cluster structure of lattice points on the sphere 6

3. Some geometric constructions 8

3.1. Background from differential geometry 8

3.2. The complexification of $\Sigma$ and the submanifolds $\Sigma(v) \quad 10$

4. Using complexification 13

4.1. The strategy 13

4.2. An oscillatory integral 14

5. A lower bound for the mean-square of $\varphi^{\mathbb{C}}(Z) e^{-2 \pi i\left\langle\xi_{0}, Z\right\rangle} \quad 19$

5.1. Representing $\varphi^{\mathbb{C}}$ on $\Sigma\left(v_{0}, \tau\right)$ by a short sum 19

5.2. Proof of the lower bound on the mean square 20

6. Non-zero curvature: Proof of Theorem $1.2 \quad 23$

6.1. The strategy

Date: November 6, 2018. 
6.2. An oscillatory integral 23

6.3. Geometric considerations 25

6.4. Vanishing of Fourier coefficients in the larger caps 26

Appendix A. The two-dimensional case: Using the ABC theorem 29

A.1. Complexification 29

A.2. A lower bound for the height of monomials 30

A.3. Proof of Theorem 1.1 32

References 33

\section{IntRoduCtion AND STATEMENT OF RESUlts}

Our goal in this paper is to study the nodal sets of high-frequency eigenfunctions on the standard flat torus $\mathbb{T}^{d}=\mathbb{R}^{d} / \mathbb{Z}^{d}$. The eigenvalues of the Laplacian on $\mathbb{T}^{d}$ are of the form $4 \pi^{2} \lambda^{2}$, with $\lambda^{2}$ an integer, with corresponding eigenfunctions which are trigonometric polynomials of the form

$$
\varphi(x)=\sum_{\substack{|\xi|=\lambda \\ \xi \in \mathbb{Z}^{d}}} a_{\xi} e^{2 \pi i\langle\xi, x\rangle}
$$

all of whose frequencies are integer points on the sphere $|x|=\lambda$. If $\lambda \neq 0$ then the mean value $\int_{\mathbb{T}^{d}} \varphi(x) d x=0$ vanishes. The nodal set is the locus of zeros $\{\varphi(x)=0\}$, which is a hypersurface (codimension one) in $\mathbb{T}^{d}$, necessarily real-analytic, possibly with singularities. We would like to study how the nodal sets vary when we increase the eigenvalue. It is known that for any real-analytic Riemannian manifold, the volume of the nodal sets is commensurable with $\lambda$ [5]. In this paper, we address a different question: As $\lambda$ grows, can a fixed hypersurface lie on infinitely many nodal sets?

1.1. Dimension $d=2$. In the flat torus in two dimensions, we can have fixed curves where many eigenfunctions vanish, as in the case the line $y=$ 0 which is on the nodal set of all the eigenfunction $\sin (2 \pi m x) \sin (2 \pi n y)$. More generally, if $\Sigma$ is a closed geodesic, one can easily construct an infinite sequence of eigenvalues with eigenfunctions vanishing on $\Sigma$. However this is essentially the only such possibility for the flat torus in two dimensions, where we can settle this problem completely:

Theorem 1.1. Let $\Sigma \subset \mathbb{T}^{2}$ be a real-analytic curve. Then a necessary and sufficient condition that there are eigenfunctions $\varphi_{\lambda}$ with arbitrarily large frequencies which vanish on $\Sigma$ is that it be a segment of a closed geodesic.

Theorem 1.1 is an easy consequence of our uniform $L^{2}$-restriction theorem [1] on the torus, which shows that for any smooth curve $\Sigma \subset \mathbb{T}^{2}$ with nowhere zero curvature, there is some $\lambda_{\Sigma}>0$ and $C_{\Sigma}>0$ so that for all eigenfunctions $\varphi_{\lambda}$ with $\lambda \geq \lambda_{\Sigma}$, we have

$$
\int_{\Sigma}\left|\varphi_{\lambda}\right|^{2} \geq C_{\Sigma}\left\|\varphi_{\lambda}\right\|^{2}
$$


The restriction lower bound (1.2) can be used to show nonvanishing on curves (Theorem 1.1) which are not segments of closed geodesics: Indeed, since the curve is real-analytic, if it is not flat it has only finitely many flat points, hence by shrinking it we may assume that it has nowhere-zero curvature, and then non-vanishing follows from the lower bound (1.2). If $\Sigma$ is flat, but not a segment of a closed geodesic, then it is a segment of an unbounded geodesic, in which case it is easy to check that no eigenfunction can vanish on it (though we do not know the restriction bound (1.2) in this case, see [14]).

We also have a completely different proof of Theorem 1.1 using an algebraic argument, relying on the "ABC theorem" of Brownawell-Masser [3] and Voloch [16] which we present in Appendix A.

1.2. Higher dimensions. Suppose now that $\Sigma \subset \mathbb{T}^{d}$ is a hypersurface (codimension one). A special role is played by flat hypersurfaces, which on the torus are affine hyperplanes. As in the two-dimensional case, if $\Sigma$ is flat and closed (a closed totally geodesic hypersurface), then it is contained in the nodal set of eigenfunctions with arbitrarily large eigenvalues, e.g. if $\Sigma=\left\{x \in \mathbb{T}^{d}:\langle\xi, x\rangle=c\right\}$ for some $\xi \in \mathbb{Z}^{d}$, then it is part of the nodal set of the eigenfunctions $\varphi_{n}(x)=\sin 2 \pi n(\langle\xi, x\rangle-c)$ for all $n \geq 1$. However, in more than two dimensions, we do have non-flat hypersurfaces (that is such that not all principal curvatures vanish) contained in the nodal set of eigenfunctions with arbitrarily large eigenvalues. For instance, let $\varphi_{0}(x, y)$ be an eigenfunction on the two-dimensional torus with eigenvalue $\lambda_{0}^{2}$, and $S_{0} \subset \mathbb{T}^{2}$ a curved segment contained in its nodal set. For $n \geq 0$ let $\varphi_{n}(x, y, z)=\varphi_{0}(x, y) \cos 2 \pi n z$, which is an eigenfunction on $\mathbb{T}^{3}$ with eigenvalue $\lambda_{n}^{2}=\lambda_{0}^{2}+n^{2}$, whose nodal set contains the cylindrical set $\Sigma=$ $S_{0} \times S^{1}$. Thus if $S_{0}$ is curved then $\Sigma$ is not flat yet lies within the nodal set of all the $\varphi_{n}$. A similar construction works to show that there are $\Sigma \subset \mathbb{T}^{d}$ which are cylindrical in the direction of any closed geodesic for which there are eigenfunctions with arbitrarily large eigenvalues vanishing on $\Sigma$.

So assume that $\Sigma$ has nowhere zero Gauss-Kronecker curvature, meaning all principal curvatures are nowhere zero (see $\S 3$ for a discussion). We then show a higher-dimensional version of Theorem 1.1;

Theorem 1.2. Let $\Sigma \subset \mathbb{T}^{d}$ be a real analytic (codimension one) hypersurface, with nowhere-vanishing Gauss-Kronecker curvature. Then there is some $\lambda_{\Sigma}>0$ so that if $\lambda \geq \lambda_{\Sigma}$, then $\Sigma$ cannot lie within the nodal set of any eigenfunction $\varphi_{\lambda}$.

A key ingredient in this result deals with any hypersurface which is not flat. As noted above, there are examples of such hypersurfaces contained in the nodal set of eigenfunctions with arbitrarily large eigenvalues. Our next result constrains the possible frequencies of such eigenfunctions, showing that the Fourier coefficients $a_{\xi}$ are negligible for frequencies $\xi$ whose directions $\xi /|\xi|$ lie outside a fixed cap on the sphere: 
Theorem 1.3. Let $\Sigma \subset \mathbb{T}^{d}$ be a real analytic hypersurface which is not flat. Then there is a cap $\Omega_{\Sigma} \subset S^{d-1}$ so that for all eigenfunctions $\varphi_{\lambda}$ which vanish on $\Sigma$ we have

$$
\left|a_{\xi}\right| \ll \frac{\left\|\varphi_{\lambda}\right\|_{2}}{\lambda^{N}}, \quad \forall N>1
$$

for all $\xi \in \mathcal{E}$ such that $\xi /|\xi| \in \Omega_{\Sigma}$.

Here and elsewhere we use the notation $f \ll g$ to mean that there is some $c>0$ so that $f \leq c g$.

1.3. About the proofs. At this time we do not have an analogue of the uniform restriction theorem (1.2) in arbitrary dimension (except dimension three [2]). We refer to [4, 9] for upper bounds in a more general context. We are also not able to use an analogue of the "abc theorem" as in $\S$, However, we retain the feature of passing to the complex domain, combined with some ideas from the $L^{2}$-restriction theorem, to prove Theorem 1.3 in arbitrary dimension. The idea is that the eigenfunctions $\varphi$ are naturally extended to holomorphic functions $\varphi^{\mathbb{C}}$ on the complexification $\mathbb{C}^{d} / \mathbb{Z}^{d}$ of $\mathbb{T}^{d}$, and since $\Sigma$ is real-analytic it too admits a complexification $\Sigma^{\mathbb{C}} \subset \mathbb{C}^{d} / \mathbb{Z}^{d}$. We then show in $\S 3$ that there is a fixed cap of directions $\Omega_{\Sigma} \subset S^{d-1}$, and $\tau=\tau_{\Sigma}>0$ depending only on $\Sigma$, so that for $v \in \Omega_{\Sigma}$, there is a submanifold $\Sigma(v, \tau) \subset \Sigma^{\mathbb{C}}$ such that for all $Z \in \Sigma(v, \tau)$ the imaginary part $\operatorname{Im} Z=t v$ is parallel to $v$, and $\tau<t<2 \tau$. For frequencies $\xi$ for which $v:=-\xi /|\xi| \in \Omega_{\Sigma}$, we give in $\S 5$ a lower bound for the $L^{2}$-restriction of $\varphi^{\mathbb{C}}(Z) e^{-2 \pi i\langle\xi, Z\rangle}$ to $\Sigma(v, \tau)$ of the form

$$
\int_{\Sigma(v, \tau)}\left|\varphi^{\mathbb{C}}(Z) e^{-2 \pi i\langle\xi, Z\rangle}\right|^{2} d \mu(Z) \gg\left|a_{\xi}\right|^{2}+O\left(\frac{1}{\lambda^{N}}\right)
$$

where $d \mu$ is a smooth measure on $\Sigma(v, \tau)$. To compare with the restriction theorem (1.2), note that in that case the RHS is $\sum_{\xi}\left|a_{\xi}\right|^{2}$. That $\Sigma$ is not flat is used to guarantee decay of certain oscillatory integrals in $\S 4.2$. On the other hand, if $\varphi$ vanishes on $\Sigma$ then its holomorphic extension $\varphi^{\mathbb{C}}$ will vanish on $\Sigma^{\mathbb{C}}$ and in particular the LHS of (1.4) will vanish. This will prove Theorem 1.3.

To get vanishing of all Fourier coefficients and hence Theorem 1.2, we need all principal curvatures to be nonzero. The extra argument needed to deduce it from Theorem 1.3 is given in $\S$ 6. In all of our results we need an arithmetic ingredient, concerning the structure of lattice points on the sphere, which is given in $\S 2$.

1.4. The sphere and Legendre polynomials. One may investigate corresponding questions for the nodal sets of eigenfunctions on other manifolds. However even in seemingly simple situations the problem is as yet open. Consider the situation on the two-dimensional sphere $S^{2} \subset \mathbb{R}^{3}$, where the Laplace-Beltrami operator has eigenvalues $n(n+1)$ with the dimension of the corresponding eigenspace $H_{n}$ being $2 n+1$. We use spherical coordinates: 
the colatitude $\theta \in[0, \pi]$ and longitude $\phi \in[0,2 \pi]$. In these coordinates, we may take as a basis of the eigenspace $H_{n}$ the spherical harmonics

$$
Y_{n}^{m}(\theta, \phi)=P_{n}^{m}(\cos \theta) e^{i m \phi}, \quad-n \leq m \leq n
$$

where $P_{n}^{m}(x)$ are Legendre functions (to get real valued functions replace $e^{i m \phi}$ by sine and cosine). In particular for $m=0$ one gets the zonal spherical functions $Y_{n}(\theta, \phi)=P_{n}(\cos \theta)$ where $P_{n}(x)$ are Legendre polynomials

$$
P_{n}(x)=\frac{1}{2^{n}} \sum_{j=0}^{\lfloor n / 2\rfloor}(-1)^{j}\left(\begin{array}{c}
n \\
j
\end{array}\right)\left(\begin{array}{c}
2 n-2 j \\
n-2 j
\end{array}\right) x^{n-2 j}
$$

which are orthogonal polynomials on the interval $[-1,1]$. The Legendre polynomial $P_{n}(x)$ has $n$ simple zeros $x_{n, j} \in[-1,1]$.

The nodal set of the zonal spherical harmonic $Y_{n}^{0}$ is the union of the parallels $\theta=\theta_{n, j}, j=1, \ldots, n$ where $x_{n, j}=\cos \theta_{n, j}$ are the zeros of the Legendre polynomial $P_{n}(x)$. Since $P_{n}(-x)=(-1)^{n} P_{n}(x)$, for odd $n$ we have $P_{n}(0)=0$, and so we find that the zonal spherical harmonics $Y_{n}^{0}$ vanish on the equator $\theta=\pi / 2$ for odd $n$.

The equator $\theta=0$ was singled out by our choice of coordinates, but by symmetry a corresponding construction works for all rotations of the equator. Thus every closed geodesic on the sphere lies within the nodal set of eigenfunctions with arbitrarily large eigenvalues, as happens on the flat torus.

A simple version of our results for the flat torus is to ask whether the other parallels (besides the equator) lie on nodal sets of infinitely many eigenfunctions. As a special case, one can conjecture that a parallel (other than the equator) cannot lie within the nodal set of more than one zonal spherical harmonic. This special case is equivalent to the conjecture of Stieltjes [15] that $P_{m}(x)$ and $P_{n}(x)$ have no common roots except $x=0$ when $m, n$ are both odd. In fact, in the same letter [15], Stieltjes put forth the stronger conjecture that $P_{2 n}(x)$ and $P_{2 n+1}(x) / x$ are irreducible. This was taken up by Holt [8] in 1912, and by Schur and his student Hildegard Ille [10], see also [17, 18]. Around 1960 irreducibility was known for all $n \leq 500$ with a few exceptions (which nowadays are easily checked by computer).

Acknowledgments: We thank Aaron Levine for his comments on Appen$\operatorname{dix} \mathrm{A}$, and the anonymous referees for their comments and suggestions. J.B. was supported in part by N.S.F. grants DMS 0808042 and DMS 0835373. Z.R. was supported by the Oswald Veblen Fund during his stay at the Institute for Advanced Study and by the Israel Science Foundation (grant No. 1083/10). 


\section{Cluster structure of lattice points on the SPhere}

For $R \geq 1$, we denote by $\mathcal{E}=\mathcal{E}_{R}=\mathbb{Z}^{d} \cap R S^{d-1}$ the set of lattice points on the sphere of radius $R$ (assuming $R^{2}$ is an integer):

$$
\mathcal{E}_{R}:=\left\{\xi \in \mathbb{Z}^{d}:|\xi|=R\right\}
$$

As is well known, the number of points in $\mathcal{E}$ satisfies $\mathcal{E}_{R} \ll R^{\epsilon}$ for all $\epsilon>0$ in dimension $d=2$, while in higher dimension $\# \mathcal{E}_{R}$ grows polynomially. Jarnik's theorem [11] places constraints on location of lattice points in small caps:

Theorem 2.1 (Jarnik's Theorem). There is some $c_{d}>0$ so that all lattice points in the cap

$$
\mathcal{E} \cap\left\{|x|=R:\left|x-x_{0}\right|<c_{d} R^{\frac{1}{d+1}}\right\}
$$

lie on an affine hyperplane.

We will need more information about the "cluster structure" of the set $\mathcal{E}$. We define recursively two sequences $c(d), \delta(d)$ with initial conditions

$$
\delta(2)<\frac{1}{3}, \quad c(2)=0
$$

and satisfying for $d \geq 3$,

$$
\begin{gathered}
c(d)=2 \max \left(c(d-1), \frac{d}{\delta(d-1)}\right) \\
\delta(d)=\frac{1}{2(d+1)(1+c(d))}
\end{gathered}
$$

Proposition 2.2. Let $\mathcal{E} \subseteq \mathbb{Z}^{d} \cap\{|x|=R\}$ be a subset of the set of lattice points on the sphere of radius $R$. If $\rho<R^{\delta(d)}$ then:

a) For any subset $\mathcal{F} \subset \mathcal{E}$, there is an overset $\mathcal{F} \subseteq \tilde{\mathcal{F}} \subset \mathcal{E}$ satisfying

$$
\begin{gathered}
\operatorname{diam}(\tilde{\mathcal{F}}) \leq \operatorname{diam}(\mathcal{F})+\rho^{1+c(d)} \\
\operatorname{dist}(\tilde{\mathcal{F}}, \mathcal{E} \backslash \tilde{\mathcal{F}})>\rho
\end{gathered}
$$

b) We may decompose $\mathcal{E}=\coprod_{\alpha} \mathcal{E}_{\alpha}$ into subsets satisfying

$$
\begin{gathered}
\operatorname{dist}\left(\mathcal{E}_{\alpha}, \mathcal{E}_{\beta}\right)>\rho, \quad \alpha \neq \beta \\
\operatorname{diam} \mathcal{E}_{\alpha}<\rho^{1+c(d)}
\end{gathered}
$$

To prove Proposition 2.2, we will need:

Lemma 2.3. If $1<\rho<R^{\delta(d)}$, and $x_{0}, \ldots, x_{K} \in \mathcal{E}$ are distinct elements satisfying

$$
\operatorname{dist}\left(x_{i}, x_{i+1}\right) \leq \rho, \quad i=0, \ldots, K-1
$$

then $K \ll \rho^{c(d)}$. 
Proof. We prove the claim by induction on the dimension $d$.

For $d=2$, we note that by Jarnik's theorem, there is some $c_{2}>0$ such that all lattice points in an arc $\left\{|x|=R:\left|x-x_{0}\right|<c_{2} R^{1 / 3}\right\}$ are co-linear, an in particular there can be at most two of them. Thus if $\rho<\frac{1}{2} c_{2} R^{1 / 3}$ then we cannot have a chain $x_{0}, x_{1}, x_{2}$ with $\operatorname{dist}\left(x_{i}, x_{i+1}\right)<\rho$ since then we would have three lattice point in cap of size $c_{2} R^{1 / 3}$.

Now let $d \geq 3$ and assume the contrary, that there is some chain $x_{0}, \ldots x_{K}$ of length $K>\rho^{c(d)}$. Let $K^{\prime}=\left\lfloor\rho^{c(d)}\right\rfloor$ and consider the initial chain $C^{\prime}=$ $\left\{x_{0}, \ldots x_{K^{\prime}}\right\}$ of length $K^{\prime}$. The diameter of this chain is at most $\operatorname{diam}\left(C^{\prime}\right) \leq$ $K^{\prime} \rho \ll \rho^{c(d)+1}<R^{\delta(d)(1+c(d))}$ and hence by (2.3) $)$, $\operatorname{diam}\left(C^{\prime}\right)<R^{1 / 2(d+1)}=$ $o\left(R^{1 /(d+1)}\right)$. Therefore by Jarnik's theorem, this subchain is contained in some hyperplane $H$, that is in the intersection of the sphere of radius $R$ with the hyperplane $H$, which is a $(d-2)$-dimensional sphere of some radius $R_{1} \ll R$.

Thus we get a $\rho$-chain of length $K^{\prime}$ in dimension $d-1$. There are two possibilities:

1) If $\rho<R_{1}^{\delta(d-1)}$ then the inductive hypothesis allows us to conclude $K^{\prime}<\rho^{c(d-1)}=o\left(\rho^{c(d)}\right)$ by (2.2) , contradicting $K^{\prime} \approx \rho^{c(d)}$.

2) If $\rho>R_{1}^{\delta(d-1)}$ then we bound the number of lattice points on a $(d-2)$ dimensional sphere of radius $R_{1}$ by $\left(1+2 R_{1}\right)^{d} \ll R_{1}^{d}$ by replacing the sphere by a $d$-dimensional cube containing the sphere (this is crude but uniform with respect to the hyperplane $H)$. Hence $K^{\prime} \ll R_{1}^{d} \ll \rho^{\frac{d}{\delta(d-1)}}$, which contradicts $K^{\prime} \approx \rho^{c(d)}$ by (2.2).

We may now prove Proposition 2.2.

Proof. We set $\mathcal{F}_{0}:=\mathcal{F}$ and define

$$
\mathcal{F}_{i}:=\mathcal{F} \cup\left\{x \in \mathcal{E}: \operatorname{dist}\left(x, \mathcal{F}_{i-1}\right) \leq \rho\right\}
$$

to be the set of lattice points at distance less than $\rho$ from the previous set. So we have an ascending sequence

$$
\mathcal{F}_{0}=\mathcal{F} \subseteq \mathcal{F}_{1} \subseteq \mathcal{F}_{2} \subseteq \ldots
$$

If $\mathcal{F}_{0}, \mathcal{F}_{1}, \ldots \mathcal{F}_{k}$ are all distinct then $k<\rho^{c(d)}$ since then we can form a chain $x_{0}, \ldots x_{k}$ of distinct elements $x_{i} \in \mathcal{F}_{i} \backslash \mathcal{F}_{i-1}$, with $\operatorname{dist}\left(x_{i}, x_{i+1}\right) \leq \rho$. Hence by Lemma 2.3 we have $k<\rho^{c(d)}$.

Thus for some $0 \leq K<\rho^{c(d)}$ we must have $\mathcal{F}_{K}=\mathcal{F}_{K+1}$. Note that if $\mathcal{F}_{K+1}=\mathcal{F}_{K}$ then $\mathcal{F}_{K+j}=\mathcal{F}_{K}$ for all $j \geq 1$ and by definition, if $y \in \mathcal{E} \backslash \mathcal{F}_{K}$ then $\operatorname{dist}\left(y, \mathcal{F}_{K}\right)>\rho$. Thus taking $\tilde{\mathcal{F}}:=\mathcal{F}_{K}$ we get a set which is well separated from its complement, that is (2.5) holds, and for any $y \in \tilde{\mathcal{F}}$ there is some $x \in \mathcal{F}$ with $\operatorname{dist}(x, y)<K \rho<\rho^{1+c(d)}$, so that (2.4) holds.

To prove the second part, we take some lattice point $x_{1} \in \mathcal{E}$ and let $\mathcal{F}=\mathcal{E} \cap \operatorname{Ball}\left(x_{1}, \rho^{1+c(d)}\right)$. Using the first part we find an overset $\mathcal{F} \subseteq \tilde{\mathcal{F}} \subseteq \mathcal{E}$ satisfying (2.5) and (2.4) and set $\mathcal{E}_{1}=\tilde{\mathcal{F}}$, so that $\operatorname{diam} \mathcal{E}_{1} \ll \rho^{1+c(d)}$ and $\operatorname{dist}\left(\mathcal{E}_{1}, \mathcal{E} \backslash \mathcal{E}_{1}\right)>\rho$. Now replace $\mathcal{E}$ by $\mathcal{E} \backslash \mathcal{E}_{1}$ and continue the process. 


\section{Some Geometric Constructions}

3.1. Background from differential geometry. Let $\Sigma \subset \mathbb{T}^{d}$ be a realanalytic hypersurface, which we assume is non-singular. We consider a small parametric patch on $\Sigma$ which we may assume looks like a graph, that is there is a real-analytic function $f\left(x_{1}, \ldots, x_{d-1}\right)$ so that

$$
\gamma(x)=(x, f(x)), \quad|x|<\delta
$$

is a parametrization of $\Sigma$.

For each point $p \in \Sigma$, denote by $T_{p} \Sigma$ the tangent space to $\Sigma$ at $p$. On $\Sigma$ we have the frame field

$$
X_{j}:=\frac{\partial \gamma}{\partial x_{j}}=(0, \ldots, \overbrace{1}^{j}, \ldots, 0, \frac{\partial f}{\partial x_{j}})
$$

which gives at each point $p$ a basis of $T_{p} \Sigma$. The general tangent vector may be given as the linear combination

$$
v=\sum_{j=1}^{d-1} w_{j} X_{j}=(\omega, \nabla f \cdot \omega), \quad \omega=\left(w_{1}, \ldots w_{d-1}\right)
$$

A choice of a unit normal field to the hypersurface $\Sigma$ at the point $p=$ $(x, f(x))$ is given by

$$
N_{p}:=\frac{1}{\sqrt{1+|\nabla f(x)|^{2}}}(-\nabla f(x), 1)
$$

The unit normal field defines the Gauss map $N: \Sigma \rightarrow S^{d-1}$. The shape operator for the hypersurface $\Sigma$, determined by the choice (3.3) of unit normal, is the linear map

$$
S_{p}: T_{p} \Sigma \rightarrow T_{p} \Sigma, \quad v \mapsto-\nabla_{v} N_{p}
$$

that is $S_{p}$ is (minus) the derivative of the Gauss map.

The shape operator is self-adjoint:

$$
\left\langle S_{p}(u), v\right\rangle=\left\langle u, S_{p}(v)\right\rangle
$$

and associated to it one has a symmetric bilinear form, the second fundamental form

$$
I I_{p}(u, v)=\left\langle S_{p}(u), v\right\rangle
$$

The coefficients of the second fundamental form with respect to the frame field $\left\{X_{j}\right\}$ may be computed explicitly in terms of the derivatives

as

$$
X_{i, j}=\frac{\partial^{2} \gamma}{\partial x_{i} \partial x_{j}}=\left(\overrightarrow{0}, \frac{\partial^{2} f}{\partial x_{i} \partial x_{j}}\right)
$$

$$
\left\langle S\left(X_{i}\right), X_{j}\right\rangle=\left\langle N, X_{i, j}\right\rangle=\frac{\frac{\partial^{2} f}{\partial x_{i} \partial x_{j}}}{\sqrt{|\nabla f|^{2}+1}}
$$


The eigenvalues of the shape operator are the principal curvatures of $\Sigma$, and the determinant of $S$ is called the Gauss-Kronecker curvature of $\Sigma$. The hypersurface $\Sigma$ is flat, i.e. is an affine hyper-plane, if and only if the unit normal $N_{p}$ is constant, which happens if and only if all principal curvatures vanish, that is the shape operator is identically zero.

Given a unit tangent vector $u \in T_{p} \Sigma$, the normal curvature of $\Sigma$ at $p$ in the direction $u$ is defined as

$$
k(u)=\left\langle S_{p}(u), u\right\rangle
$$

If we cut the hypersurface $\Sigma$ by the plane spanned by $u$ and the unit normal $N_{p}$, we get a curve whose tangent at $p$ is the vector $u$, and whose curvature at $p$ is $k(u)$. For any nonzero tangent vector $v$ given as in (3.2), the normal curvature in direction $v$ is

$$
k(v)=\left\langle S\left(\frac{v}{|v|}\right), \frac{v}{|v|}\right\rangle=\frac{1}{\sqrt{|\nabla f|^{2}+1}} \frac{\omega^{T} D_{x, x} f \omega}{|\omega|^{2}+(\nabla f \cdot \omega)^{2}}
$$

where $D_{x, x} f=\left(\frac{\partial^{2} f}{\partial x_{i} \partial x_{j}}\right)$ is the Hessian matrix of $f$.

Directions for which the normal curvature vanishes are called asymptotic directions. Thus a tangent vector $v$ as in (3.2) points in an asymptotic direction if and only if

$$
\omega^{T} D_{x, x} f \omega=0
$$

The set of asymptotic directions at a point $p$ is called the asymptotic cone. The hypersurface $\Sigma$ is flat at the point $p$ if and only if every direction is asymptotic, that is the asymptotic cone coincides with the whole tangent space.

Lemma 3.1. Suppose $\Sigma$ is not flat. Then after shrinking $\Sigma$, we can find a cap $\Omega_{\Sigma} \subset S^{d-1}$ of directions $v=\left(\omega, w_{d}\right)$ so that:

i) There is a point $p=\gamma(x) \in \Sigma$ so that $v$ is tangent to $\Sigma$ at $p$, equivalently satisfies

$$
\nabla f(x) \cdot \omega=w_{d}
$$

ii) The direction $v$ is not an asymptotic direction for all $p \in \Sigma$, that is for all $x$ and all $v=\left(\omega, w_{d}\right) \in \Omega_{\Sigma}$ we have

$$
\omega^{T} D_{x x} f(x) \omega \neq 0
$$

Proof. Since $\Sigma$ is not flat, the Hessian $D_{x, x} f$ is not identically zero (if it were, $f(x)=a+b \cdot x$ would be linear hence $\Sigma$ would be flat). Then we may assume by further shrinking $\Sigma$ that in fact the Hessian matrix $D_{x, x} f(x) \neq 0$ is nonzero for all $|x|<\delta$.

Since $D_{x, x} f(0)$ is not the zero matrix, the asymptotic cone

$$
\left\{\omega \in \mathbb{R}^{d-1}: \omega^{T} D_{x, x} f(0) \omega=0\right\}
$$


has lower dimension and hence there is an open cone of directions for which $\omega^{T} D_{x, x} f(0) \omega \neq 0$. Moreover, by continuity of $x \mapsto D_{x, x} f(x)$, we get some $\delta>0$ and an open cone $\mathcal{C}$ so that

$$
\omega^{T} D_{x, x} f(x) \omega \neq 0, \quad \forall|x|<\delta, \quad \forall \omega \in \mathcal{C}
$$

Consider the map

$$
\begin{aligned}
V: \operatorname{Ball}(\overrightarrow{0}, \delta) \times \mathcal{C} & \rightarrow \mathbb{R}^{d} \\
(x, \omega) & \mapsto v=\sum_{j=1}^{d-1} w_{j} X_{j}=(\omega, \nabla f(x) \cdot \omega)
\end{aligned}
$$

We claim that the map $V$ is a submersion, i.e. the Jacobian of $V$ has maximal rank for each $(x, \omega)$, hence the image of $V$ contains an open set of directions $\Omega_{\Sigma} \subset S^{d-1}$.

To see this, compute the Jacobian of $V$ :

$$
D_{x, \omega} V=\left(\frac{\partial V}{\partial \omega_{i}}, \frac{\partial V}{\partial x_{j}}\right)=\left(\begin{array}{cc}
I_{d-1} & 0_{d-1} \\
\nabla f(x) & D_{x, x} f(x) \cdot \omega
\end{array}\right)
$$

Since $\omega^{T} D_{x, x} f(x) \omega \neq 0$ for all $x$ and $\omega \in \mathcal{C}$, hence $D_{x, x} f(x) \cdot \omega \neq \overrightarrow{0}$, it follows that the rank of $D_{x, \omega} V$ is $d$ as claimed.

Thus for each unit vector $v=\left(\omega, w_{d}\right) \in \Omega_{\Sigma}$, there is a point $p=\gamma(x) \in \Sigma$ so that $v$ is tangent to $\Sigma$ at $p$, equivalently is orthogonal to the normal, so satisfies

$$
\nabla f(x) \cdot \omega=w_{d}
$$

Moreover, for all such $x$ we have $\omega^{T} D_{x, x} f(x) \omega \neq 0$.

Note that if $v$ satisfies (3.10), (3.11) then so does $-v$.

3.2. The complexification of $\Sigma$ and the submanifolds $\Sigma(v)$. Since $f$ is real-analytic, there is a holomorphic extension $F(z)$ of $f$ to some neighborhood $\mathcal{U} \subset \mathbb{C}^{d-1}$. This gives a holomorphic extension of the parametrization

$$
\gamma^{\mathbb{C}}: z \in \mathcal{U} \mapsto(z, F(z))
$$

and we define the image

$$
\Sigma^{\mathbb{C}}:=\left\{\gamma^{\mathbb{C}}(x+i y)=(z, F(z)), z \in \mathcal{U}\right\}
$$

to be the holomorphic extension of the surface $\Sigma$.

Let $v \in S^{d-1}$ be a unit vector in the cap guaranteed by Lemma 3.1, so there is some $p=\left(x_{0}, f\left(x_{0}\right)\right) \in \Sigma$ with $v \perp N_{p}$. For such $v$, we will define a submanifold $\Sigma(v) \subset \Sigma^{\mathbb{C}}$ so that

- If $Z \in \Sigma(v)$ then $\operatorname{Im} Z=t v$ is parallel to $v$.

- $\Sigma(v) \cap \Sigma \subseteq\left\{p \in \Sigma: N_{p} \perp v\right\}$, i.e. at the real points $p$ of $\Sigma(v)$, the normal vector $N_{p}$ is orthogonal to $v$, equivalently $v$ is tangent to $\Sigma$ at $p$. 
To do so, write $v=\left(\omega, w_{d}\right)$ and note that the vector-valued function $\operatorname{Im} \gamma^{\mathbb{C}}(x+$ $i t \omega)-t \vec{v}$ is real-analytic in $t$ and vanishes at $t=0$. Hence we can write

$$
\operatorname{Im} \gamma^{\mathbb{C}}(x+i t \omega)-t \vec{v}=t(\overrightarrow{0}, h(x, t))
$$

with $h(x, t)=h_{v}(x, t)$ real analytic. We want to define $\Sigma(v)$ by the vanishing of $h(x, t)$. To do so, we need:

Lemma 3.2. For $v=\left(\omega, w_{d}\right) \in \Omega_{\Sigma}$, let $p=\left(x_{0}, f\left(x_{0}\right)\right) \in \Sigma$ be such that $v \in T_{p} \Sigma$. Then

$$
h\left(x_{0}, 0\right)=0
$$

and

$$
\nabla h\left(x_{0}, 0\right)=\left(D_{x, x} f\left(x_{0}\right) \omega, 0\right) \neq \overrightarrow{0}
$$

Proof. Start at a point $p=\left(x_{0}, f\left(x_{0}\right)\right) \in \Sigma$ with $N_{p} \perp v$. To find the value of the function $h(x, t)$ at $t=0$, expand

$$
\operatorname{Im} F(x, t \omega)=\operatorname{Im} F(x, 0)+t \nabla_{y} \operatorname{Im} F(x, 0) \cdot \omega+O\left(t^{2}\right)
$$

By the Cauchy-Riemann equations, $\nabla_{y} \operatorname{Im} F(x, 0)=\nabla_{x} \operatorname{Re} F(x, 0)=\nabla f(x)$ and since $\operatorname{Im} F(x, 0)=0$ we find

$$
h(x, 0)=\lim _{t \rightarrow 0} \frac{\operatorname{Im} F(x, t \omega)}{t}-w_{d}=\nabla f(x) \cdot \omega-w_{d}
$$

Since the normal direction to the surface $\Sigma$ at $p$ is given by the vector $\left(-\nabla f\left(x_{0}\right), 1\right)$, we find that if $N_{p} \perp v$ then $h\left(x_{0}, 0\right)=0$.

To show (3.20), note that since $f(x)$ is real-analytic, its holomorphic extension $F$ satisfies $\overline{F(z)}=F(\bar{z})$, that is

$$
\operatorname{Re} F(\bar{z})=\operatorname{Re} F(z), \quad \operatorname{Im} F(\bar{z})=-\operatorname{Im} F(z)
$$

showing that $\operatorname{Im} F(x+i t \omega)$ is odd in $t$, hence $h(x, t)$ is even in $t$. Therefore we have

$$
\frac{\partial h}{\partial t}(x, 0)=0
$$

Moreover since $\operatorname{Im} F(x, t \omega)$ is odd in $t$,

$$
\operatorname{Im} F(x, t \omega)=t\left(\nabla_{y} \operatorname{Im} F\right)(x, 0) \cdot \omega+O\left(t^{3}\right)
$$

and hence

$$
\nabla_{x} \frac{\operatorname{Im} F(x, t \omega)}{t}=D_{x y} \operatorname{Im} F(x, 0) \cdot \omega+O\left(t^{2}\right)
$$

By Cauchy-Riemann, $D_{x y} \operatorname{Im} F\left(x_{0}, 0\right)=D_{x x} \operatorname{Re} F\left(x_{0}, 0\right)=D_{x x} f\left(x_{0}\right)$ and hence we find

$$
\nabla_{x} h\left(x_{0}\right)=D_{x x} f\left(x_{0}\right) \cdot \omega
$$

proving (3.20).

By construction of the cap $\Omega_{\Sigma}$ in Lemma 3.1, we know that $\omega^{T} D_{x, x} f(x) \omega \neq$ 0 for all $x$; in particular $D_{x x}(f)\left(x_{0}\right) \cdot \omega \neq \overrightarrow{0}$ and therefore $\nabla h\left(x_{0}, 0\right) \neq \overrightarrow{0}$. 
Since $\nabla_{x} h\left(x_{0}, 0\right)=D_{x, x} f\left(x_{0}\right) \omega \neq \overrightarrow{0}$, we may use the Implicit Function Theorem to guarantee that there is a neighborhood of $\left(x_{0}, 0\right)$ in the $(x, t)$ domain where $\nabla_{x} h(x, t) \neq \overrightarrow{0}$ and the condition $h(x, t)=0$ defines a smooth $(d-1)$-dimensional submanifold. After shrinking $\Sigma$ and relabeling, we may then assume that for all $(x, t)$,

$$
\frac{\partial h}{\partial x_{1}}(x, t) \neq 0
$$

Using the Implicit Function Theorem, we can then write

$$
x_{1}=x_{1}(t, \widehat{x}), \quad \widehat{x}:=\left(x_{2}, \ldots, x_{d-1}\right)
$$

We then define

$$
\Sigma(v):=\left\{\gamma^{\mathbb{C}}\left(\left(x_{1}(t, \widehat{x}), \widehat{x}\right)+i t \omega\right):|t|<\delta,|\widehat{x}|<\delta\right\}
$$

Note that since $v$ varies in a compact set, we may choose $\delta>0$ to work uniformly for all such $v$. Hence for $\tau \ll \delta, \Sigma(v)$ contains the set

$$
\Sigma(v, \tau):=\left\{Z=\gamma^{\mathbb{C}}\left(\left(x_{1}(t, \widehat{x}), \widehat{x}\right)+i t \omega\right) \in \Sigma(v): \tau<t<2 \tau,|\widehat{x}|<\delta\right\}
$$

so that $Z \in \Sigma(v, \tau)$ implies that $\operatorname{Im} Z=t v$, with $t \in(\tau, 2 \tau)$.

We define a smooth measure $d \mu$ on $\Sigma(v, \tau)$ by taking a smooth bump function $\psi(t, \widehat{x})$, supported in $t \in[\tau, 2 \tau]$, and setting

$$
\int_{\Sigma(v, \tau)} g(Z) d \mu(Z):=\int_{\tau<t<2 \tau} \int_{|\widehat{x}|<\delta} g\left(\gamma^{\mathbb{C}}\left(\left(x_{1}(t, \widehat{x}), \widehat{x}\right)+i t \omega\right)\right) \psi(t, \widehat{x}) d t d \widehat{x}
$$

We will restrict $\psi$ by requiring that its support is disjoint from the lowerdimensional set of $(t, \widehat{x})$ satisfying

$$
\begin{aligned}
& \left\langle\left(\nabla_{x} \operatorname{Re} F\right)(x, t \omega), \omega\right\rangle=w_{d} \\
& \left\langle\left(\nabla_{y} \operatorname{Re} F\right)(x, t \omega), \omega\right\rangle=0
\end{aligned}
$$

This condition will be used in $\S 4.2$ to ensure decay of an oscillatory integral. 


\section{USING COMPLEXIFICATION}

We start with an eigenfunction of the Laplacian with eigenvalue $\lambda^{2}$

$$
\varphi_{\lambda}(\vec{X})=\sum_{\xi \in \mathcal{E}} a_{\xi} e^{2 \pi i\langle\xi, \vec{X}\rangle}, \quad \vec{X} \in \mathbb{T}^{d}
$$

which we normalize by

$$
\sum_{\xi}\left|a_{\xi}\right|^{2}=1
$$

We want to show that if $\Sigma$ has nowhere zero Gauss-Kronecker curvature, then for $\lambda>\lambda_{\Sigma}, \varphi_{\lambda}$ cannot vanish on the fixed hypersurface $\Sigma$. We proceed to do so by showing initially that the Fourier coefficients $a_{\xi}$ are negligible for all frequencies whose directions $\xi /|\xi|$ lie in a cap $\Omega_{\Sigma}$ depending only on $\Sigma$. All that is required for this is that $\Sigma$ not be flat:

Theorem 4.1. Assume $\Sigma \subset \mathbb{T}^{d}$ is not flat. Then there is a cap $\Omega_{\Sigma} \subset S^{d-1}$ so that for all eigenfunctions $\varphi_{\lambda}$ which vanish on $\Sigma$ we have

$$
\left|a_{\xi}\right| \ll \frac{1}{\lambda^{N}}, \quad \forall N>1
$$

for all $\xi \in \mathcal{E}$ such that $-\xi /|\xi| \in \Omega_{\Sigma}$.

4.1. The strategy. Fix $\xi_{0} \in \mathcal{E}$ so that

$$
v_{0}=-\frac{\xi_{0}}{\left|\xi_{0}\right|} \in \Omega_{\Sigma}
$$

lies in the set of directions guaranteed by Lemma 3.1.

We have a holomorphic extension $\varphi^{\mathbb{C}}(\vec{Z})$ of $\varphi$ by replacing $\vec{X}$ by $\vec{Z}=$ $\vec{X}+i \vec{Y} \in \mathbb{C}^{d}$, which is a function on $\mathbb{C}^{d} / \mathbb{Z}^{d}$. We will give a lower bound for the mean square of $\varphi^{\mathbb{C}}(Z) e^{-2 \pi i\left\langle\xi_{0}, Z\right\rangle}$ restricted to the submanifold $\Sigma\left(v_{0}, \tau\right)$ :

$$
\int_{\Sigma\left(v_{0}, \tau\right)}\left|\varphi^{\mathbb{C}}(Z) e^{-2 \pi i\left\langle\xi_{0}, Z\right\rangle}\right|^{2} d \mu(Z) \gg\left|a_{\xi_{0}}\right|^{2}+O\left(\frac{1}{\lambda^{N}}\right)
$$

where $d \mu$ is the smooth measure on $\Sigma\left(v_{0}\right)$ constructed in (3.27).

On the other hand, vanishing of $\varphi$ on $\Sigma$ implies vanishing of $\varphi^{\mathbb{C}}(\vec{Z})$ on the holomorphic extension $\Sigma^{\mathbb{C}}$ of our surface $\Sigma$. In particular, the mean square of $\varphi^{\mathbb{C}}(Z) e^{-2 \pi i\left\langle\xi_{0}, Z\right\rangle}$ along $\Sigma\left(v_{0}, \tau\right)$ vanishes:

$$
\int_{\Sigma\left(v_{0}, \tau\right)}\left|\varphi^{\mathbb{C}}(Z) e^{-2 \pi i\left\langle\xi_{0}, Z\right\rangle}\right|^{2} d \mu(Z)=0
$$

and combining with the lower bound (4.5), this will prove Theorem 4.1.

To prove the lower bound on the mean square (4.5), we show that on $\Sigma\left(v_{0}, \tau\right)$ we may represent $\varphi^{\mathbb{C}}(Z) e^{-2 \pi i\left\langle\xi_{0}, Z\right\rangle}$ up to negligible error by a sum over frequencies in a small cap:

$$
\varphi^{\mathbb{C}}(\vec{Z}) e^{-2 \pi i\left\langle\xi_{0}, \vec{Z}\right\rangle}=\sum_{\mathcal{E}^{\prime}} a_{\xi} e^{2 \pi i\left\langle\xi-\xi_{0}, Z\right\rangle}+O\left(\frac{1}{\lambda^{N}}\right), \quad Z \in \Sigma\left(v_{0}, \tau\right)
$$


where the sum is over a certain set $\mathcal{E}^{\prime}$ of frequencies contained in a cap of size $\approx \sqrt{\lambda} \log \lambda$ around $\xi_{0}$.

On squaring out the sum in we will be faced with oscillatory integrals of the form

$$
J_{\xi, \xi^{\prime}}:=\int_{\Sigma\left(v_{0}, \tau\right)} e^{2 \pi i\left(\left\langle\xi-\xi_{0}, Z\right\rangle-\left\langle\xi^{\prime}-\xi_{0}, \bar{Z}\right\rangle\right)} d \mu(Z)
$$

We will bound these integrals by

$$
J_{\xi, \xi^{\prime}} \ll \frac{1}{\left|\xi-\xi^{\prime}\right|^{N}}, \quad \xi \neq \xi^{\prime} \in \mathcal{E}^{\prime}
$$

Here the fact that $\Sigma$ is not flat is crucial. Armed with this estimate, we will prove (4.5) by using the cluster structure of the set of frequencies $\mathcal{E}$ shown in $\S 2$.

4.2. An oscillatory integral. We want to bound the oscillatory integral $J_{\xi, \xi^{\prime}}$ in (4.7), or writing out explicitly,

$$
J_{\xi, \xi^{\prime}}=\int e^{2 \pi i\left|\xi-\xi^{\prime}\right| \phi_{u}(t, \widehat{x})} \mathcal{A}_{\xi, \xi^{\prime}}(t, \widehat{x}) d t d \widehat{x}
$$

where we write $u=\frac{\xi-\xi^{\prime}}{\left|\xi-\xi^{\prime}\right|}$ and for any vector $u=\left(u_{1}, \ldots, u_{d}\right)$ the phase function $\phi_{u}$ is defined by

$$
\begin{aligned}
\phi_{u}(t, \widehat{x}) & =\langle u, G(t, \widehat{x})\rangle=\langle u,(x, \operatorname{Re} F(x, t \omega))\rangle \\
\widehat{x} & =\left(x_{2}, \ldots, x_{d-1}\right), \quad x=\left(x_{1}, \widehat{x}\right) \\
G(t, \widehat{x}) & =\operatorname{Re} \gamma^{\mathbb{C}}(x+i t \omega)=(x, \operatorname{Re} F(x, t \omega))
\end{aligned}
$$

and with amplitude

$$
\begin{gathered}
\mathcal{A}_{\xi, \xi^{\prime}}(t, \widehat{x})=e^{-2 \pi t\left(A(\xi)+A\left(\xi^{\prime}\right)\right)} \psi(t, \widehat{x}) \\
A(\xi)=\left\langle\xi-\xi_{0}, v_{0}\right\rangle
\end{gathered}
$$

and $\psi(t, \widehat{x})$ is a bump function. The region of integration in the $(t, \widehat{x})$ domain is a small ball such that $\tau<t<2 \tau$.

Lemma 4.2. Let $v_{0}=\left(\omega, w_{d}\right)$ be as given in (4.4). Then for all unit vectors $u$ orthogonal to $v_{0}$, the phase function $\phi_{u}(t, \widehat{x})$ is non constant, and the stationary points of $\phi_{u}$ lie on a subset of lower dimension, which is independent of $u$, namely the points $(t, \widehat{x})$ where

$$
\begin{aligned}
& \left\langle\left(\nabla_{x} \operatorname{Re} F\right)(x, t \omega), \omega\right\rangle=w_{d} \\
& \left\langle\left(\nabla_{y} \operatorname{Re} F\right)(x, t \omega), \omega\right\rangle=0
\end{aligned}
$$

Proof. Write out the phase function explicitly as

$$
\phi_{u}(t, \widehat{x})=u_{1} x_{1}(t, \widehat{x})+\sum_{j=2}^{d-1} u_{j} x_{j}+u_{d} \operatorname{Re} F\left(x_{1}, \widehat{x}, t \omega\right)
$$


Assume first that

$$
\frac{\partial x_{1}}{\partial t} \neq 0
$$

on the domain of integration.

We first dispose of the possibility that $u_{d}=0$. In that case,

$$
\phi_{u}(t, \widehat{x})=u_{1} x_{1}(t, \widehat{x})+\sum_{j=2}^{d-1} u_{j} x_{j}
$$

At a stationary point,

$$
0=\frac{\partial \phi_{u}}{\partial t}=u_{1} \frac{\partial x_{1}}{\partial t}
$$

and since $\frac{\partial x_{1}}{\partial t} \neq 0$ on the support of $\psi$, we find $u_{1}=0$ so that $u=$ $\left(0, u_{2}, \ldots, u_{d-1}, 0\right)$ and $\phi_{u}=\sum_{j=2}^{d-1} u_{j} x_{j}$ is linear, $\nabla \phi_{u}=u$ and $\phi_{u}$ has no stationary points.

Assume from now that $u_{d} \neq 0$. Consider the differential operator

$$
\mathcal{L}=\frac{w_{1}}{\frac{\partial x_{1}}{\partial t}} \frac{\partial}{\partial t}+\sum_{j=2}^{d-1} w_{j}\left(\frac{\partial}{\partial x_{j}}-\frac{\frac{\partial x_{1}}{\partial x_{j}}}{\frac{\partial x_{1}}{\partial t}} \frac{\partial}{\partial t}\right)=A \frac{\partial}{\partial t}+\sum_{j=2}^{d-1} w_{j} \frac{\partial}{\partial x_{j}}
$$

with

$$
A=\frac{1}{\frac{\partial x_{1}}{\partial t}}\left(w_{1}-\sum_{j=2}^{d-1} w_{j} \frac{\partial x_{1}}{\partial x_{j}}\right)
$$

A calculation using the chain rule shows that for a function of the form $H(x, t \omega)$ we have

$$
\mathcal{L}\{H(x, t \omega)\}=\left\langle\nabla_{x} H, \omega\right\rangle+A\left\langle\nabla_{y} H, \omega\right\rangle
$$

Applying $\mathcal{L}$ to the phase function $\phi_{u}$, using $\mathcal{L} x_{j}=w_{j}$, gives

$$
\mathcal{L} \phi_{u}=\sum_{j=1}^{d-1} u_{j} w_{j}+u_{d}\left(\left\langle\nabla_{x} \operatorname{Re} F, \omega\right\rangle+A\left\langle\nabla_{y} \operatorname{Re} F, \omega\right\rangle\right)
$$

Hence at a stationary point, where $\mathcal{L} \phi_{u}=0$, we find on using the orthogonality of $u$ and $v_{0}$, that

$$
u_{d}\left(\left\langle\nabla_{x} \operatorname{Re} F, \omega\right\rangle+A\left(\left\langle\nabla_{y} \operatorname{Re} F, \omega\right\rangle\right)=-\sum_{j=1}^{d-1} u_{j} w_{j}=u_{d} w_{d}\right.
$$

and since $u_{d} \neq 0$ we get

$$
\left\langle\nabla_{x} \operatorname{Re} F, \omega\right\rangle+A\left\langle\nabla_{y} \operatorname{Re} F, \omega\right\rangle=w_{d}
$$

Likewise, applying $\mathcal{L}$ to the relation $\operatorname{Im} F(x, t \omega)=t w_{d}$ we get on using $\mathcal{L} t=A$ that

$$
\left\langle\nabla_{x} \operatorname{Im} F, \omega\right\rangle+A\left\langle\nabla_{y} \operatorname{Im} F, \omega\right\rangle=A w_{d}
$$


Applying the Cauchy-Riemann equations $\nabla_{x} \operatorname{Im} F=-\nabla_{y} \operatorname{Re} F, \nabla_{y} \operatorname{Im} F=$ $\nabla_{x} \operatorname{Re} F$ gives

$$
A\left\langle\nabla_{x} \operatorname{Re} F, \omega\right\rangle-\left\langle\nabla_{y} \operatorname{Re} F, \omega\right\rangle=A w_{d}
$$

The unique solution of the system (4.21), (4.23) is then

$$
\left\langle\nabla_{x} \operatorname{Re} F, \omega\right\rangle=w_{d}, \quad\left\langle\nabla_{y} \operatorname{Re} F, \omega\right\rangle=0
$$

Now assume that $\phi_{u}$ is constant, so that (4.24) holds for all $t, \widehat{x}$. Then we may apply the differential operator $\mathcal{L}$ to (4.24) to get

$$
\mathcal{L}\left\langle\nabla_{x} \operatorname{Re} F, \omega\right\rangle=\omega^{T} \nabla_{x, x} \operatorname{Re} F \omega+A \omega^{T} \nabla_{x, y} \operatorname{Re} F \omega=0
$$

and

$$
\mathcal{L}\left\langle\nabla_{y} \operatorname{Re} F, \omega\right\rangle=\omega^{T} \nabla_{x, y} \operatorname{Re} F \omega+A \omega^{T} \nabla_{y, y} \operatorname{Re} F \omega=0
$$

By the Cauchy-Riemann equations, $\nabla_{y, y} \operatorname{Re} F=-\nabla_{x, x} \operatorname{Re} F$ and we find

$$
\omega^{T} \nabla_{x, y} \operatorname{Re} F \omega-A \omega^{T} \nabla_{x, x} \operatorname{Re} F \omega=0
$$

The unique solution of the system (4.25), (4.27) is

$$
\omega^{T} \nabla_{x, x} \operatorname{Re} F \omega=0=\omega^{T} \nabla_{x, y} \operatorname{Re} F \omega
$$

which assuming that $\phi_{u}$ is constant on $\Sigma\left(v_{0}\right)$, holds throughout $\Sigma\left(v_{0}\right)$ and in particular on the real locus $t=0$, where $\operatorname{Re} F(x, 0)=f(x)$, where we find

$$
\omega^{T} \nabla_{x, x} f(x) \omega=0, \quad \forall x \in \Sigma\left(v_{0}\right) \cap \Sigma
$$

This contradicts (3.11), that is that $v_{0}$ is not an asymptotic direction at any point on $\Sigma$. Thus $\phi_{u}$ is non-constant, and being real-analytic its stationary points (where $\nabla \phi_{u}=\overrightarrow{0}$ ) lie on a lower-dimensional subset Crit $(u)$ of $\Sigma\left(v_{0}\right)$.

Since (4.13) holds at the stationary points, which is independent of $u$, $\operatorname{Crit}(u)$ are confined to lie inside a lower-dimensional subset which is independent of $u$.

Next consider the case $\frac{\partial x_{1}}{\partial t} \equiv 0$ on the support of $\psi$. We claim that still (4.13) holds.

We first dispose of the case $u_{d}=0$ when $\phi_{u}=u_{1} x_{1}+\sum_{j=2}^{d-1} u_{j} x_{j}$ with $u \cdot w=\sum_{j=1}^{d-1} u_{j} w_{j}=0$. (We leave the case $d=2$ as an exercise). If $u_{1}=0$ then $\phi_{u}$ is a non-zero linear function and has no stationary points. Otherwise, at a stationary point,

$$
0=\frac{\partial \phi_{u}}{\partial x_{j}}=u_{1} \frac{\partial x_{1}}{\partial x_{j}}+u_{j}
$$

Differentiating the relation $\operatorname{Im} F(x, t \omega)=t w_{d}$ with respect to $x_{j}$ gives

$$
0=\frac{\partial x_{1}}{\partial x_{j}} \frac{\partial \operatorname{Im} F}{\partial x_{1}}+\frac{\partial \operatorname{Im} F}{\partial x_{j}}=-\frac{\partial x_{1}}{\partial x_{j}} \frac{\partial \operatorname{Re} F}{\partial y_{1}}-\frac{\partial \operatorname{Re} F}{\partial y_{j}}
$$


by the Cauchy-Riemann equations. Hence

$$
\begin{aligned}
\left\langle\nabla_{y} \operatorname{Re} F, \omega\right\rangle & =w_{1} \frac{\partial \operatorname{Re} F}{\partial y_{1}}+\sum_{j=2}^{d-1} w_{j} \frac{\partial \operatorname{Re} F}{\partial y_{j}} \\
& =w_{1} \frac{\partial \operatorname{Re} F}{\partial y_{1}}-\sum_{j=2}^{d-1} w_{j} \frac{\partial x_{1}}{\partial x_{j}} \frac{\partial \operatorname{Re} F}{\partial y_{1}} \\
& =\frac{\partial \operatorname{Re} F}{\partial y_{1}}\left(w_{1}+\sum_{j=2}^{d-1} w_{j} \frac{u_{j}}{u_{1}}\right)=0
\end{aligned}
$$

since by orthogonality of $u$ and $v_{0}$ and vanishing of $u_{d}$, we have $w_{1}+$ $\sum_{j=2}^{d-1} w_{j} \frac{u_{j}}{u_{1}}=0$.

Assume now that $u_{d} \neq 0$. Then at a stationary point,

$$
0=\frac{\partial \phi_{u}}{\partial t}=u_{d}\left\langle\nabla_{y} \operatorname{Re} F, \omega\right\rangle
$$

and $u_{d} \neq 0$ implies $\left\langle\nabla_{y} \operatorname{Re} F, \omega\right\rangle=0$. Differentiating the relation $\operatorname{Im} F(x, t \omega)=$ $t w_{d}$ with respect to $t$, using independence of $x_{1}$ relative to $t$, gives

$$
w_{d}=\left\langle\nabla_{y} \operatorname{Im} F, \omega\right\rangle=\left\langle\nabla_{x} \operatorname{Re} F, \omega\right\rangle
$$

by the Cauchy-Riemann equations. Thus in all cases (4.13) hold at a stationary point.

Now differentiate the relation $\left\langle\nabla_{y} \operatorname{Re} F(x, t \omega), \omega\right\rangle=0$ with respect to $t$, keeping in mind that $x_{1}$ is independent of $t$, to get $\omega^{T} \nabla_{y, y} \operatorname{Re} F \omega=0$ and using the Cauchy-Riemann equation we get $\omega^{T} \nabla_{x, x} \operatorname{Re} F \omega=0$. Specializing to the real locus $t=0$ again gives a contradiction.

Lemma 4.3. Let $v_{0}$ be as in (4.4), and set $D=(\log \lambda)^{2}$. Then for all $\xi \neq \xi^{\prime}$ lying in a cap of size $\sqrt{\lambda D}$ around $\lambda v_{0}$ we have

$$
\left|J_{\xi, \xi^{\prime}}\right| \ll \frac{1}{\left|\xi-\xi^{\prime}\right|^{r}}, \quad \forall r \geq 1
$$

Proof. Write

$$
J_{\xi, \xi^{\prime}}=\int e^{2 \pi i\left|\xi-\xi^{\prime}\right| \Phi_{\xi, \xi^{\prime}}(t, \widehat{x})} \mathcal{A}_{\xi, \xi^{\prime}}(t, \widehat{x}) d t d \widehat{x} d x
$$

where

$$
\Phi_{\xi, \xi^{\prime}}(t, \widehat{x}):=\left\langle\frac{\xi-\xi^{\prime}}{\left|\xi-\xi^{\prime}\right|}, G(t, \widehat{x})\right\rangle
$$

We claim that there is some $C>0$ for which for all $\xi \neq \xi^{\prime}$ in our cap, the phase functions satisfy

$$
\left\|\nabla \Phi_{\xi, \xi^{\prime}}(t, \widehat{x})\right\| \geq C
$$

Indeed, decompose $\xi-\xi^{\prime}$ into components along $v_{0}$ and orthogonal to it:

$$
\xi-\xi^{\prime}=k u+\left\langle\xi-\xi^{\prime}, v_{0}\right\rangle v_{0}, \quad u \perp v_{0}, \quad\|u\|=1
$$


Since $\xi, \xi^{\prime}$ lie in a cap of size $\sqrt{\lambda D}$ on the sphere of radius $\lambda$, the difference $\xi-\xi^{\prime}$ is almost orthogonal to $v_{0}$ and we claim that

$$
\left|\left\langle\frac{\xi-\xi^{\prime}}{\left|\xi-\xi^{\prime}\right|}, v_{0}\right\rangle\right| \ll \frac{\sqrt{\lambda D}}{\lambda}=o(1)
$$

Indeed, writing $\xi=\xi_{0}+\eta, \xi^{\prime}=\xi_{0}+\eta^{\prime}$, with $|\eta|,\left|\eta^{\prime}\right| \leq \sqrt{\lambda D}$ we get

$$
2\left\langle\eta, \xi_{0}\right\rangle+|\eta|^{2}=0=2\left\langle\eta^{\prime}, \xi_{0}\right\rangle+\left|\eta^{\prime}\right|^{2}
$$

and since $\xi_{0}=-\lambda v_{0}$,

$$
\left|\left\langle\frac{\xi-\xi^{\prime}}{\left|\xi-\xi^{\prime}\right|}, v_{0}\right\rangle\right|=\left|\left\langle\frac{\eta-\eta^{\prime}}{\left|\eta-\eta^{\prime}\right|}, v_{0}\right\rangle\right|=\left|\frac{|\eta|^{2}-\left|\eta^{\prime}\right|^{2}}{2 \lambda\left|\eta-\eta^{\prime}\right|}\right| \leq \frac{|\eta|+\left|\eta^{\prime}\right|}{2 \lambda}=\frac{\sqrt{\lambda D}}{\lambda}
$$

as claimed. Likewise we have

$$
A(\xi), A\left(\xi^{\prime}\right) \leq \frac{D}{2}
$$

because

Therefore we find

$$
A(\xi)=\left\langle\eta, v_{0}\right\rangle=-\left\langle\eta, \frac{\xi_{0}}{\lambda}\right\rangle=\frac{|\eta|^{2}}{2 \lambda} \leq \frac{D}{2}
$$

$$
|k| \sim\left|\xi-\xi^{\prime}\right|
$$

Thus

$$
\Phi_{\xi, \xi^{\prime}}(t, \widehat{x})=\frac{k}{\left|\xi-\xi^{\prime}\right|} \phi_{u}(t, \widehat{x})+o(1)\left\langle v_{0}, G(t, \widehat{x})\right\rangle
$$

By our choice (3.28) of $\psi$ and as a consequence of Lemma 4.2, we know that $\phi_{u}$ has no critical point in $\operatorname{supp} \psi$ for all $u \perp v_{0}$ and so there is some $C>0$ so that $\left|\nabla \phi_{u}(t, \widehat{x})\right|>2 C$ for all $u \perp v_{0}$ and all $(t, \widehat{x}) \in \operatorname{supp} \psi$. Therefore (recalling that $|k| \sim\left|\xi-\xi^{\prime}\right|$ ) for $\lambda \gg 1$ we have

$$
\left|\nabla \Phi_{\xi, \xi^{\prime}}(t, \widehat{x})\right|>C
$$

as claimed.

Integrating by parts we get that

$$
J_{\xi, \xi^{\prime}} \ll\left\|\mathcal{A}_{\xi, \xi^{\prime}}\right\|_{C^{r}} \frac{1}{\left|\xi-\xi^{\prime}\right|^{r}}, \quad \forall r \geq 1
$$

Since $0 \leq A(\xi), A\left(\xi^{\prime}\right) \leq D$, and $\tau \leq t \leq 2 \tau$ on $\operatorname{supp} \psi$, we may bound the $C^{r}$-norm of the amplitude function $\mathcal{A}_{\xi, \xi^{\prime}}=e^{-2 \pi t\left(A(\xi)+A\left(\xi^{\prime}\right)\right)} \psi(t, \widehat{x})$ by

$$
\left\|\mathcal{A}_{\xi, \xi^{\prime}}\right\|_{C^{r}} \ll_{\psi}\left(1+A(\xi)+A\left(\xi^{\prime}\right)\right)^{r+1} e^{-2 \pi \tau\left(A(\xi)+A\left(\xi^{\prime}\right)\right)}=O(1)
$$

(the implied constant depends only on $\psi, \tau$ and $r$, not on $\xi, \xi^{\prime}$ ), which gives the required estimate. 
5. A LOWER BOUNd FOR THE MEAN-SQUARE OF $\varphi^{\mathbb{C}}(Z) e^{-2 \pi i\left\langle\xi_{0}, Z\right\rangle}$

5.1. Representing $\varphi^{\mathbb{C}}$ on $\Sigma\left(v_{0}, \tau\right)$ by a short sum. We show that for $Z \in \Sigma\left(v_{0}, \tau\right)$ we may represent $\varphi^{\mathbb{C}}(\vec{Z}) e^{-2 \pi i\left\langle\xi_{0}, \vec{Z}\right\rangle}$ by the part of its Fourier expansion whose frequencies lie in a small cap around $\xi_{0}$, up to a negligible error.

For $\xi \in \mathcal{E}$, set

$$
A(\xi)=\left\langle\xi-\xi_{0}, v_{0}\right\rangle
$$

Observe that since all vectors $\xi \in \mathcal{E}$ lie on a sphere, and thus no two vectors can lie on the same positive ray, we have $\left\langle\xi, \xi_{0}\right\rangle\left\langle\left\langle\xi_{0}, \xi_{0}\right\rangle\right.$ for all vectors $\xi \neq \xi_{0}$ and hence

$$
A(\xi)>0, \quad \xi \neq \xi_{0}, \quad A\left(\xi_{0}\right)=0
$$

Let

$$
D \approx(\log \lambda)^{2}
$$

The set

$$
\mathcal{E}^{\prime}:=\{\xi \in \mathcal{E}: A(\xi)<D\}
$$

is contained in a cap of size $\approx \sqrt{\lambda D}$ centered at $\xi_{0}$. Note that for $\xi$ in this cap, $\xi-\xi_{0}$ is almost perpendicular to $v_{0}$.

Lemma 5.1. For $\vec{Z}=\vec{X}+i \vec{Y} \in \Sigma\left(v_{0}, \tau\right)$, we have

$$
\varphi^{\mathbb{C}}(\vec{Z}) e^{-2 \pi i\left\langle\xi_{0}, \vec{Z}\right\rangle}=\sum_{A(\xi) \leq D} a_{\xi} e^{2 \pi i\left\langle\xi-\xi_{0}, \vec{X}\right\rangle} e^{-2 \pi t A(\xi)}+O\left(\frac{1}{\lambda^{N}}\right)
$$

for all $N \geq 1$.

Proof. We define a subset $T\left(v_{0}\right) \subset \mathbb{C}^{d} / \mathbb{Z}^{d}$ by

$$
T\left(v_{0}\right):=\left\{\vec{Z} \in \mathbb{C}^{d}: \operatorname{Im} \vec{Z}=|\operatorname{Im} \vec{Z}| v_{0}\right\}
$$

that is the complex vectors whose imaginary parts point along the ray in the direction of $v_{0}$. Restricting $\varphi^{\mathbb{C}}$ to $T\left(v_{0}\right)$, we have

$$
\varphi^{\mathbb{C}}(\vec{Z}) e^{-2 \pi i\left\langle\xi_{0}, \vec{Z}\right\rangle}=\sum_{\xi} a_{\xi} e^{2 \pi i\left\langle\xi-\xi_{0}, \vec{X}\right\rangle} e^{-2 \pi t A(\xi)}, \quad t:=|\operatorname{Im} \vec{Z}|
$$

Now restrict $\vec{Z}$ further by assuming that it lies in the set

$$
T\left(v_{0} ; \tau\right):=\left\{\vec{Z} \in T\left(v_{0}\right): \tau<|\operatorname{Im} \vec{Z}|<2 \tau\right\}
$$

Then for $\vec{Z} \in T\left(v_{0} ; \tau\right)$ we have

$$
\begin{aligned}
\left|\sum_{A(\xi)>D} a_{\xi} e^{2 \pi i\left\langle\xi-\xi_{0}, \vec{Z}\right\rangle}\right| \leq \sum_{A(\xi)>D}\left|a_{\xi}\right| e^{-2 \pi|\operatorname{Im} \vec{Z}| D} & \\
& \ll(\# \mathcal{E})^{1 / 2} e^{-2 \pi \tau D} \ll \frac{1}{\lambda^{N}}, \quad \forall N>1
\end{aligned}
$$

using $\sum_{\xi}\left|a_{\xi}\right|^{2}=1, \# \mathcal{E} \ll \lambda^{d-2+\epsilon}$ and $A(\xi) \geq D,|\operatorname{Im} \vec{Z}|>\tau$. 
Hence for $\vec{Z} \in T\left(v_{0} ; \tau\right)$ we have

$$
\varphi^{\mathbb{C}}(\vec{Z}) e^{-2 \pi i\left\langle\xi_{0}, \vec{Z}\right\rangle}=\sum_{A(\xi) \leq D} a_{\xi} e^{2 \pi i\left\langle\xi-\xi_{0}, \vec{X}\right\rangle} e^{-2 \pi t A(\xi)}+O\left(\frac{1}{\lambda^{N}}\right)
$$

In particular, since $\Sigma\left(v_{0}, \tau\right) \subset T\left(v_{0}, \tau\right)$ we proved (5.5).

5.2. Proof of the lower bound on the mean square. We now want to prove the lower bound (4.5) for the mean square, namely that

$$
\int_{\Sigma\left(v_{0}, \tau\right)}\left|\varphi^{\mathbb{C}}(Z) e^{-2 \pi i\left\langle\xi_{0}, Z\right\rangle}\right|^{2} d \mu(Z) \gg\left|a_{\xi_{0}}\right|^{2}+O\left(\frac{1}{\lambda^{N}}\right)
$$

Hence what we need follows from the following Lemma, once we recall that $A(\xi) \geq 0$ for all $\xi \in \mathcal{E}^{\prime}$ and that $A\left(\xi_{0}\right)=0$ :

Proposition 5.2. There is some $C>0$ so that

$$
\int_{\Sigma\left(v_{0}, \tau\right)}\left|\sum_{\xi \in \mathcal{E}^{\prime}} a_{\xi} e^{2 \pi i\left\langle\xi-\xi_{0}, Z\right\rangle}\right|^{2} d \mu(Z) \geq C \sum_{\xi \in \mathcal{E}^{\prime}}\left|a_{\xi}\right|^{2} e^{-8 \pi \tau A(\xi)}+O\left(\frac{1}{\lambda^{N}}\right)
$$

for all $N>1$.

Proof. We note that for $Z \in \Sigma\left(v_{0}, \tau\right)$,

$$
\left\langle\xi-\xi_{0}, Z\right\rangle=\left\langle\xi-\xi_{0}, G(t, \widehat{x})\right\rangle+i t A(\xi)
$$

where

$$
G(t, \widehat{x})=\operatorname{Re} \gamma^{\mathbb{C}}\left(\left(x_{1}, \widehat{x}\right)+i t \omega\right)=\left(x_{1}, \widehat{x}, \operatorname{Re} F\left(\left(x_{1}, \widehat{x}\right)+i t \omega\right)\right)
$$

and so we need to show that

$$
\int\left|\sum_{\xi \in \mathcal{E}^{\prime}} a_{\xi} e^{-2 \pi t A(\xi)} e^{2 \pi i\left\langle\xi-\xi_{0}, G(t, \widehat{x})\right\rangle}\right|^{2} d \mu(t, \widehat{x}) \geq C \sum_{\xi \in \mathcal{E}^{\prime}}\left|a_{\xi}\right|^{2} e^{-8 \pi \tau A(\xi)}+O\left(\frac{1}{\lambda^{N}}\right)
$$

for all $N>1$, where $d \mu(t, \widehat{x})=\psi(t, \widehat{x}) d t d \widehat{x}$.

Let $d\left(\mathcal{E}^{\prime}\right)$ be the minimal dimension of an affine hyperplane which contains all the frequencies $\mathcal{E}^{\prime}$, so $d\left(\mathcal{E}^{\prime}\right) \leq d$. We show by induction on $d^{\prime}=d\left(\mathcal{E}^{\prime}\right)$ that (5.12) holds.

The case $d^{\prime}=1$ : This means all frequencies lie on a line, and hence (since they lie on a sphere) there are at most two of them. If there is exactly one frequency the claim is clear, so we need to treat the case $\mathcal{E}^{\prime}=\left\{\xi, \xi^{\prime}\right\}$ consists of two distinct frequencies. That is we want to show that

$$
\begin{aligned}
\int \mid a_{\xi} e^{2 \pi i\left\langle\xi-\xi_{0}, G(t, \widehat{x})\right\rangle} e^{-2 \pi t A(\xi)} & +\left.a_{\xi^{\prime}} e^{2 \pi i\left\langle\xi^{\prime}-\xi_{0}, G(t, \widehat{x})\right\rangle} e^{-2 \pi t A\left(\xi^{\prime}\right)}\right|^{2} d \mu \\
& \geq C\left(\left|a_{\xi}\right|^{2} e^{-8 \pi \tau A(\xi)}+\left|a_{\xi^{\prime}}\right|^{2} e^{-8 \pi \tau A\left(\xi^{\prime}\right)}\right)
\end{aligned}
$$

Write

$$
\mathcal{A}_{\xi}(t)=\left|a_{\xi}\right| e^{-2 \pi t A(\xi)}, \quad a_{\xi}=\left|a_{\xi}\right| e^{2 \pi i \alpha_{\xi}}
$$


Then we want to give a lower bound for

$$
\int\left|\mathcal{A}_{\xi}(t) e^{2 \pi i\left(\alpha_{\xi}+\left\langle\xi-\xi_{0}, G(t, \widehat{x})\right\rangle\right)}+\mathcal{A}_{\xi^{\prime}}(t) e^{2 \pi i\left(\alpha_{\xi^{\prime}}+\left\langle\xi^{\prime}-\xi_{0}, G(t, \widehat{x})\right\rangle\right)}\right|^{2} d \mu
$$

We have

$$
\begin{aligned}
& \left|\mathcal{A}_{\xi}(t) e^{2 \pi i\left(\alpha_{\xi}+\left\langle\xi-\xi_{0}, G(t, \widehat{x})\right\rangle\right)}+\mathcal{A}_{\xi^{\prime}}(t) e^{2 \pi i\left(\alpha_{\xi^{\prime}}+\left\langle\xi^{\prime}-\xi_{0}, G(t, \widehat{x})\right\rangle\right)}\right|^{2} \\
& =\mathcal{A}_{\xi}(t)^{2}+\mathcal{A}_{\xi^{\prime}}(t)^{2}+2 \mathcal{A}_{\xi}(t) \mathcal{A}_{\xi^{\prime}}(t) \cos 2 \pi \phi(t, \widehat{x})
\end{aligned}
$$

where the phase function is

$$
\phi(t, \widehat{x})=\alpha_{\xi}-\alpha_{\xi^{\prime}}+\left|\xi-\xi^{\prime}\right| \Phi_{\xi, \xi^{\prime}}(t, \widehat{x}), \quad \Phi_{\xi, \xi^{\prime}}(t, \widehat{x})=\left\langle\frac{\xi-\xi^{\prime}}{\left|\xi-\xi^{\prime}\right|}, G(t, \widehat{x})\right\rangle
$$

Let

$$
\mathcal{S}_{\delta}=\{(t, \widehat{x}) \in \operatorname{supp} \psi: \cos 2 \pi \phi(t, \widehat{x}) \geq-1+\delta\}
$$

According to (4.36),

$$
|\nabla \phi(t, \widehat{x})| \geq\left|\xi-\xi^{\prime}\right| C \geq C
$$

for all $(t, \widehat{x}) \in \operatorname{supp} \psi$ and all $\xi \neq \xi^{\prime} \in \mathcal{E}^{\prime}$ (using integrality for $\left|\xi-\xi^{\prime}\right| \geq 1$ ). Therefore since the phase varies by at least a fixed amount, there is some $\delta>0$ and $C>0$ (independent of $\xi, \xi^{\prime}$ ) so that

$$
\int_{\mathcal{S}_{\delta}} d \mu \geq \frac{C}{\delta}
$$

On the set $\mathcal{S}_{\delta}$ we have

$$
\begin{aligned}
\mathcal{A}_{\xi}(t)^{2}+\mathcal{A}_{\xi^{\prime}}(t)^{2}+2 \mathcal{A}_{\xi}(t) \mathcal{A}_{\xi^{\prime}}(t) \cos 2 \pi \phi(t, \widehat{x}) & \\
& \geq \mathcal{A}_{\xi}(t)^{2}+\mathcal{A}_{\xi^{\prime}}(t)^{2}+(-1+\delta) 2 \mathcal{A}_{\xi}(t) \mathcal{A}_{\xi^{\prime}}(t) \\
=\delta\left(\mathcal{A}_{\xi}(t)^{2}+\mathcal{A}_{\xi^{\prime}}(t)^{2}\right) & +(1-\delta)\left|\mathcal{A}_{\xi}(t)-\mathcal{A}_{\xi^{\prime}}(t)\right|^{2} \\
& \geq \delta\left(\left|a_{\xi}\right|^{2} e^{-8 \pi \tau A(\xi)}+\left|a_{\xi^{\prime}}\right|^{2} e^{-8 \pi \tau A\left(\xi^{\prime}\right)}\right)
\end{aligned}
$$

Therefore we find that

$$
\begin{aligned}
\text { (5.14) } & =\int\left\{\mathcal{A}_{\xi}(t)^{2}+\mathcal{A}_{\xi^{\prime}}(t)^{2}+2 \mathcal{A}_{\xi}(t) \mathcal{A}_{\xi^{\prime}}(t) \cos 2 \pi \phi(t, \widehat{x})\right\} d \mu \\
& \geq \int_{\mathcal{S}_{\delta}}\left\{\mathcal{A}_{\xi}(t)^{2}+\mathcal{A}_{\xi^{\prime}}(t)^{2}+2 \mathcal{A}_{\xi}(t) \mathcal{A}_{\xi^{\prime}}(t) \cos 2 \pi \phi(t, \widehat{x})\right\} d \mu \\
& \geq \delta\left(\left|a_{\xi}\right|^{2} e^{-8 \pi \tau A(\xi)}+\left|a_{\xi^{\prime}}\right|^{2} e^{-8 \pi \tau A\left(\xi^{\prime}\right)}\right) \int_{\mathcal{S}_{\delta}} d \mu \\
& \geq C\left(\left|a_{\xi}\right|^{2} e^{-8 \pi \tau A(\xi)}+\left|a_{\xi^{\prime}}\right|^{2} e^{-8 \pi \tau A\left(\xi^{\prime}\right)}\right)
\end{aligned}
$$

as claimed.

The case $d^{\prime} \geq 2$ : By Proposition 2.2 we may partition $\mathcal{E}^{\prime}=\coprod \mathcal{E}_{\alpha}$ where

$$
\operatorname{diam} \mathcal{E}_{\alpha} \ll \lambda^{\frac{1}{d+1}}, \quad \operatorname{dist}\left(\mathcal{E}_{\alpha}, \mathcal{E}_{\beta}\right) \gg \lambda^{\frac{1}{(d+1)(1+c(d))}}, \alpha \neq \beta
$$


Then

$$
\begin{aligned}
& \int\left|\sum_{\xi \in \mathcal{E}^{\prime}} a_{\xi} e^{2 \pi i\left\langle\xi-\xi_{0}, G(t, \widehat{x})\right\rangle} e^{-2 \pi t A(\xi)}\right|^{2} d \mu \\
& =\sum_{\alpha} \int\left|\sum_{\xi \in \mathcal{E}_{\alpha}} a_{\xi} e^{2 \pi i\left\langle\xi-\xi_{0}, G(t, \widehat{x})\right\rangle} e^{-2 \pi t A(\xi)}\right|^{2} d \mu+\sum_{\alpha \neq \beta} \sum_{\xi \in \mathcal{E}_{\alpha}} \sum_{\xi^{\prime} \in \mathcal{E}_{\beta}} a_{\xi} \overline{a_{\xi^{\prime}}} J_{\xi, \xi^{\prime}}
\end{aligned}
$$

where the oscillatory integral $J_{\xi, \xi^{\prime}}$ is given in (4.9). By Lemma 4.3 we have

$$
\left|J_{\xi, \xi^{\prime}}\right| \ll \frac{1}{\left|\xi-\xi^{\prime}\right|^{r}} \ll \frac{1}{\lambda^{N}}, \quad \forall N>1
$$

since $\left|\xi-\xi^{\prime}\right| \gg \lambda^{\frac{1}{(d+1)(1+c(d))}}$ for $\xi \in \mathcal{E}_{\alpha}, \xi^{\prime} \in \mathcal{E}_{\beta}$ with $\alpha \neq \beta$. Hence we have an upper bound for the off-diagonal terms

$$
\sum_{\alpha \neq \beta} \sum_{\xi \in \mathcal{E}_{\alpha}} \sum_{\xi^{\prime} \in \mathcal{E}_{\beta}} a_{\xi} \overline{a_{\xi^{\prime}}} J_{\xi, \xi^{\prime}} \ll \frac{1}{\lambda^{N}} \sum_{\xi \in \mathcal{E}^{\prime}}\left|a_{\xi}\right|^{2} \leq \frac{1}{\lambda^{N}}, \quad \forall N>1
$$

taking into account the normalization $\sum_{\xi}\left|a_{\xi}\right|^{2}=1$.

We now want to derive a lower bound for the diagonal terms. By Jarnik's theorem, since $\operatorname{diam} \mathcal{E}_{\alpha} \ll \lambda^{\frac{1}{d+1}}$, the set $\mathcal{E}_{\alpha}$ is contained in an affine hyperplane $H_{\alpha}$ and hence $d\left(\mathcal{E}_{\alpha}\right) \leq d-1$. Thus by the induction hypothesis we have

$$
\int\left|\sum_{\xi \in \mathcal{E}_{\alpha}} a_{\xi} e^{2 \pi i\left\langle\xi-\xi_{0}, G(t, \widehat{x})\right\rangle} e^{-2 \pi t A(\xi)}\right|^{2} d \mu \geq C \sum_{\xi \in \mathcal{E}_{\alpha}}\left|a_{\xi}\right|^{2} e^{-8 \pi \tau A(\xi)}+O\left(\frac{1}{\lambda^{N}}\right)
$$

Combining with the upper bound (5.21) for the off-diagonal terms and summing over $\alpha$ we get the required lower bound (5.12). 


\section{Non-Zero Curvature: Proof of Theorem 1.2}

Assume now that the hypersurface $\Sigma$ has nowhere zero Gauss-Kronecker curvature, i.e. all principal curvatures are nowhere zero. (The condition that $\Sigma$ is not flat means that at least one of the principal curvatures is nonzero). We will use Theorem 4.1 to prove Theorem 1.2, namely that for $\lambda>\lambda_{\Sigma}$, an eigenfunction $\varphi_{\lambda}$ cannot vanish identically on $\Sigma$.

6.1. The strategy. We keep the normalization $\sum_{\xi}\left|a_{\xi}\right|^{2}=1$. We assume that there is some cap

$$
\Omega_{0}=\operatorname{Cap}\left(w_{0}, \theta_{0}\right) \subset S^{d-1}
$$

around $w_{0}$, with opening angle $\theta$ of size $O(1)$ so that

$$
\left|a_{\xi}\right|<\frac{1}{\lambda^{N}}, \quad \forall \frac{\xi}{|\xi|} \in \Omega_{0}
$$

guaranteed by Theorem 4.1. We shall call such frequencies "negligible".

We aim to show that there is a larger cap

$$
\Omega_{1}=\operatorname{Cap}\left(w_{1}, \theta_{1}\right) \subset S^{d-1}
$$

for which all frequencies $\mathcal{E}_{1}:=\lambda \Omega_{1} \cap \mathcal{E}$ in direction $\Omega_{1}$ are negligible. Here "larger" means that say

$$
\theta_{1} \geq \theta_{0}+\delta_{0}
$$

for some fixed $\delta_{0}>0$ (independent of $\lambda$ ). We will show that all frequencies in $\mathcal{E}_{1}$ are "negligible". Proceeding in this way we will eventually show that all frequencies are "negligible", contradicting $\sum_{\xi}\left|a_{\xi}\right|^{2}=1$.

6.2. An oscillatory integral. Since $\Sigma$ has non-vanishing curvature, the unit normals to $\Sigma$ sweep out at least a cap $\operatorname{Cap}\left(u_{0}, \delta_{1}\right)$ for some $\delta_{1}>0$. If $\delta_{0}<\delta_{1} / 2$, then for any $u \in \operatorname{Cap}\left(u_{0}, \frac{\delta_{1}}{2}\right)$ we have

$$
\operatorname{Cap}\left(u, \delta_{0}\right) \subset \operatorname{Cap}\left(u_{0}, \delta_{1}\right)
$$

We choose $\delta_{0}$ sufficiently small so that for any such $u$, we have a patch $\Sigma_{u} \subset \Sigma$ on the surface so that the Gauss map

$$
N: \Sigma_{u} \rightarrow \operatorname{Cap}\left(u, \delta_{0}\right)
$$

is a diffeomorphism.

Fix a bump function $\psi$ supported in the cap $\operatorname{Cap}\left(u_{0}, \delta_{0}\right)$, from which we get a smooth measure $d \mu$ on the cap; applying rotations give smooth measure on any cap $\operatorname{Cap}\left(u, \delta_{0}\right)$, and pulling back to the patch $\Sigma_{u}$ via the Gauss map $N$ we get a smooth measure $\mu_{u}$ on $\Sigma_{u}$, which depends in a bounded way on $u \in \operatorname{Cap}\left(u_{0}, \delta_{1} / 2\right)$. Denote by

$$
\widehat{\mu}_{u}(\xi):=\int_{\Sigma_{u}} e^{-2 \pi i\langle\xi, x\rangle} d \mu_{u}(x)
$$

its Fourier transform. 
Fourier transforms of surface-carried measure are known to decay polynomially in the presence or curvature [7, 6], in fact if the surface is not flat [12. However there is faster decay in directions which are disjoint from the image of the Gauss map. We use this to prove:

Lemma 6.1. For all vectors $y \neq 0$ which do not lie in the direction of the bigger cap $\operatorname{Cap}\left(u, 2 \delta_{0}\right)$, we have

$$
\widehat{\mu}(y) \ll_{N} \frac{1}{|y|^{N}}, \quad \frac{y}{|y|} \notin \operatorname{Cap}\left(u, 2 \delta_{0}\right), \quad \forall N>0
$$

where the implied constants can be taken uniform in $u \in \operatorname{Cap}\left(u_{0}, \delta_{1} / 2\right)$

Proof. We take a regular parametrization $X: t=\left(t_{1}, \ldots, t_{d-1}\right) \mapsto X(t)$ of the patch $\Sigma_{u}$. Then the Fourier transform becomes

$$
\widehat{\mu}_{u}(y)=\int_{\mathbb{R}^{d-1}} e^{-2 \pi i|y| \phi(t)} \Psi(t) d t
$$

for a suitable amplitude $\Psi \in C_{c}^{\infty}\left(\mathbb{R}^{d-1}\right)$ and with phase function

$$
\phi(t)=\langle\widehat{y}, X(t)\rangle, \quad \widehat{y}:=\frac{y}{|y|}
$$

Our claim will follow by integration by parts if we give a uniform lower bound for the gradient of the phase function

$$
|\nabla \phi| \geq C>0
$$

The gradient of the phase function is given by

$$
\nabla \phi=D X(t) \widehat{y}
$$

where we think of $\widehat{y} \in S^{d-1}$ as a column vector and the derivative $D X=$ $\left(\frac{\partial X_{i}}{\partial t_{j}}\right)$ is a $(d-1) \times d$ matrix. Choose a row vector $\omega \in \mathbb{R}^{d-1}$ for which $\omega D X(t)$ is the orthogonal projection $P(\widehat{y})$ of $\widehat{y}$ on the tangent space $T_{X(t)} \Sigma$. Thus

$$
\omega \nabla \phi(t)=\omega D X(t) \widehat{y}=P(\widehat{y}) \cdot \widehat{y}
$$

Let $\alpha$ be the angle between the unit normal $N_{X(t)}$ and $\widehat{y}$. Then $\alpha>\delta_{0}$ since by assumption $N_{X(t)} \in \operatorname{Cap}\left(u, \delta_{0}\right)$ while $\widehat{y} \notin \operatorname{Cap}\left(u, 2 \delta_{0}\right)$. Therefore

$$
|P(\widehat{y})|=|\widehat{y}| \sin \alpha=\sin \alpha \geq \sin \delta_{0}
$$

and therefore

$$
\omega \nabla \phi(t)=P(\widehat{y}) \cdot \widehat{y}=|P(\widehat{y})|^{2} \geq\left(\sin \delta_{0}\right)^{2}
$$

On the other hand,

and so we find

$$
|\omega \nabla \phi(t)| \leq|\omega||\nabla \phi(t)|
$$

$$
|\nabla \phi| \geq \frac{\left(\sin \delta_{0}\right)^{2}}{|\omega|}
$$

and it remains to give an upper bound for $|\omega|$.

We have

$$
|P \widehat{y}|^{2}=|\omega D X|^{2}=\omega D X D X^{T} \omega^{T}
$$


Now $P(\widehat{y})$ has length at most $|\widehat{y}|=1$, being the orthogonal projection of the unit vector $\widehat{y}$, and so we find

$$
1 \geq \omega D X(t) D X(t)^{T} \omega^{T}
$$

The rows of $D X(t)$ are linearly independent since we assume that $X$ is a regular parametrization. Hence the quadratic form $D X(t) D X(t)^{T}$ is positive definite (it is the first fundamental form of the hypersurface) and so

$$
\omega D X(t) D X(t)^{T} \omega^{T} \geq c(t)|\omega|^{2}
$$

for some $c(t)>0$, and taking $c:=\min \{c(t)\}>0$ we find $1 \geq c|\omega|^{2}$, that is

$$
|\omega| \leq \frac{1}{\sqrt{c}}
$$

Thus

$$
|\nabla \phi| \geq \frac{\left(\sin \delta_{0}\right)^{2}}{\sqrt{c}}
$$

giving the required lower bound.

6.3. Geometric considerations. For a unit vector $u \in S^{d-1}$ let

$$
\tau_{u}(x)=x-2\langle x, u\rangle u
$$

be the reflection in the hyperplane orthogonal to $u$.

Fix $u_{0} \in S^{d-1}$, and $\delta>0$. Then there is some $\epsilon=\epsilon_{d}(\delta)>0$ so that for every $w \in S^{d-1}$, the set of reflected points $\tau_{u} w$, for $u$ ranging over all points in the cap $\operatorname{Cap}\left(u_{0}, \delta\right)$, contains a cap $\operatorname{Cap}\left(w_{1}, \epsilon\right)$ :

$$
\forall w \quad \exists w_{1} \text { such that } \operatorname{Cap}\left(w_{1}, \epsilon\right) \subseteq\left\{\tau_{u} w: u \in \operatorname{Cap}\left(u_{0}, \delta\right)\right\}
$$

By symmetry, $\epsilon$ is independent of the base point $u_{0}$, and depends only on the dimension $d$ and on $\delta$.

Now let $u_{0} \in S^{d-1}, \delta_{1}>0$ be as in $\S 6.2$. We fix $\delta_{0}>0$, with $\delta_{0}<\delta_{1} / 2$ sufficiently small so that the Gauss map gives a diffeomorphism (6.5), and Lemma 6.1 holds. In addition we require

$$
\delta_{0}<\frac{1}{6} \epsilon_{d}\left(\frac{\delta_{1}}{2}\right)
$$

Recall that $\operatorname{Cap}\left(w_{0}, \theta_{0}\right)=\Omega_{0}$ is a cap where we assume the Fourier coefficients $a_{\xi} \approx 0$ are negligible for all frequencies with $\xi /|\xi| \in \Omega_{0}$.

Lemma 6.2. Let $u \in \operatorname{Cap}\left(u_{0}, \delta_{1} / 2\right)$ and $B \subset \tau_{u} \operatorname{Cap}\left(w_{0}, \theta_{0}-4 \delta_{0}\right)$. Then for all unit vectors $y \notin B$, either $y \in \Omega_{0}=\operatorname{Cap}\left(w_{0}, \theta_{0}\right)$ or else

$$
\frac{x-y}{|x-y|} \notin \operatorname{Cap}\left(u, 2 \delta_{0}\right), \quad \forall x \in \tau_{u} \operatorname{Cap}\left(w_{0}, \theta_{0}-4 \delta_{0}\right), x \neq y
$$

Proof. Let $y \in S^{d-1} \backslash B$ and assume that (6.13) fails, that is there is some $x \in \tau_{u} \operatorname{Cap}\left(w_{0}, \theta_{0}-4 \delta_{0}\right)$ and $u_{1} \in \operatorname{Cap}\left(u, 2 \delta_{0}\right)$ so that

$$
y=x-|x-y| u_{1}
$$

We then need to show that $y \in \Omega_{0}$. 
The condition (6.14) means that

$$
y=\tau_{u_{1}}(x)
$$

This is because $y$ lies on the intersection of the sphere with the line through $x \in S^{d-1}$ in the direction of $u_{1}$; that intersection contains (at most) two points, one of them being $x$, which we assume is distinct from $y$. Clearly the reflection $\tau_{u_{1}}(x)$ also has this properties, so that $y=\tau_{u_{1}}(x)$. Hence we find that

$$
y=\tau_{u_{1}} x \in \tau_{u_{1}} \circ \tau_{u} \operatorname{Cap}\left(w_{0}, \theta_{0}-4 \delta_{0}\right)
$$

The composition of two distinct reflections $\tau_{u} \circ \tau_{u_{1}}$ is a rotation in the plane spanned by the two vectors $u, u_{1}$ (assumed not to be co-linear) by an angle which is twice the angle $\alpha$ between the two vectors. In our case, since $u_{1} \in \operatorname{Cap}\left(u, 2 \delta_{0}\right)$ lies in cap centered at $u$, we have $\alpha \leq 2 \delta_{0}$. Hence

$$
\tau_{u_{1}} \circ \tau_{u} \operatorname{Cap}\left(w_{0}, \theta_{0}-4 \delta_{0}\right) \subseteq \operatorname{Cap}\left(w_{0}, \theta_{0}-4 \delta_{0}+2 \alpha\right) \subseteq \operatorname{Cap}\left(w_{0}, \theta_{0}\right)
$$

so that $y \in \Omega_{0}$ as claimed.

6.4. Vanishing of Fourier coefficients in the larger caps. For each $u \in \operatorname{Cap}\left(u_{0}, \delta_{1} / 2\right)$, apply the reflection $\tau_{u}$ to the cap $\operatorname{Cap}\left(w_{0}, \theta_{0}-5 \delta_{0}\right)$ to get a cap

$$
\Omega_{1}(u):=\tau_{u} \operatorname{Cap}\left(w_{0}, \theta_{0}-5 \delta_{0}\right)=\operatorname{Cap}\left(\tau_{u} w_{0}, \theta_{0}-5 \delta_{0}\right)
$$

We now claim that the Fourier coefficients $a_{\xi}$ for frequencies whose directions lie in the cap $\Omega_{1}(u)$ are negligible:

Proposition 6.3. If $\xi /|\xi| \in \Omega_{1}(u)$ then

$$
\left|a_{\xi}\right| \ll \frac{1}{\lambda^{N}}, \quad \forall N \geq 1
$$

Proof. Let

$$
\mathcal{F}=\lambda \Omega_{1}(u) \cap \mathcal{E}
$$

and use Proposition 2.2 with $\rho=\lambda^{\delta(d)}$ to get an overset $\tilde{\mathcal{F}}, \mathcal{F} \subseteq \tilde{\mathcal{F}} \subset \mathcal{E}$ so that

$$
\operatorname{dist}(\tilde{\mathcal{F}}, \mathcal{E} \backslash \tilde{\mathcal{F}})>\lambda^{\delta(d)}
$$

and

$$
\operatorname{diam} \tilde{\mathcal{F}} \leq \operatorname{diam} \mathcal{F}+\lambda^{\frac{1}{2(d+1)}} \leq \lambda \operatorname{diam} \Omega_{1}(u)\left\{1+O\left(\lambda^{-1+\frac{1}{2(d+1)}}\right)\right\}
$$

Since $\frac{1}{\lambda} \tilde{\mathcal{F}} \supset \frac{1}{\lambda} \mathcal{F} \subset \Omega_{1}(u)=\tau_{u} \operatorname{Cap}\left(w_{0}, \theta_{0}-5 \delta_{0}\right.$ ), condition (6.20) implies that for $\lambda$ sufficiently large,

$$
\frac{1}{\lambda} \tilde{\mathcal{F}} \subset \tau_{u} \operatorname{Cap}\left(w_{0}, \theta_{0}-4 \delta_{0}\right)
$$

Set

$$
\mathcal{E}_{1}(u)=\tilde{\mathcal{F}}
$$


so that

$$
\operatorname{dist}\left(\mathcal{E}_{1}(u), \mathcal{E} \backslash \mathcal{E}_{1}(u)\right)>\lambda^{\delta(d)}
$$

and

$$
\frac{1}{\lambda} \mathcal{E}_{1}(u) \subset \tau_{u} \operatorname{Cap}\left(w_{0}, \theta_{0}-4 \delta_{0}\right)
$$

Consider the integral

$$
0=\int_{\Sigma_{u}} \overline{\varphi(x)} \sum_{\xi \in \mathcal{E}_{1}(u)} a_{\xi} e^{2 \pi i\langle\xi, x\rangle} d \mu_{u}(x)
$$

which equals zero, since we assume $\varphi=0$ on $\Sigma$.

On the other hand, expanding

$$
\varphi=\sum_{\xi \in \mathcal{E}_{1}(u)} a_{\xi} e^{2 \pi i\langle\xi, x\rangle}+\sum_{\xi \notin \mathcal{E}_{1}(u)} a_{\xi} e^{2 \pi i\langle\xi, x\rangle}
$$

gives a sum of "diagonal" and "off-diagonal" terms:

$$
\begin{aligned}
0 & =\int_{\Sigma_{u}}\left|\sum_{\xi \in \mathcal{E}_{1}(u)} a_{\xi} e^{2 \pi i\langle\xi, x\rangle}\right|^{2} d \mu_{u}(x)+\sum_{\xi \in \mathcal{E}_{1}(u)} \sum_{\eta \notin \mathcal{E}_{1}(u)} a_{\xi} \overline{a_{\eta}} \widehat{\mu}_{u}(\eta-\xi) \\
& =\text { diagonal + off-diagonal }
\end{aligned}
$$

The diagonal term can be bounded from below:

$$
\int_{\Sigma_{u}}\left|\sum_{\xi \in \mathcal{E}_{1}(u)} a_{\xi} e^{2 \pi i\langle\xi, x\rangle}\right|^{2} d \mu_{u}(x) \geq C \sum_{\xi \in \mathcal{E}_{1}(u)}\left|a_{\xi}\right|^{2}
$$

by arguing as in Proposition 5.2 (in fact by using it in the special case $A(\xi)=0)$.

We will show that the off-diagonal part is "negligible" which will give the required upper bound (6.17). To do so, decompose the off-diagonal term as

$$
\begin{aligned}
\text { off-diagonal } & =\sum_{\xi \in \mathcal{E}_{1}(u)} \sum_{\eta \in \mathcal{E}_{0} \backslash \mathcal{E}_{1}(u)} a_{\xi} \overline{a_{\eta}} \widehat{\mu}_{u}(\eta-\xi) \\
& +\sum_{\xi \in \mathcal{E}_{1}(u)} \sum_{\eta \in \mathcal{E} \backslash\left(\mathcal{E}_{0} \cup \mathcal{E}_{1}(u)\right)} a_{\xi} \overline{a_{\eta}} \widehat{\mu}_{u}(\eta-\xi)
\end{aligned}
$$

The first term is negligible because all the coefficients $a_{\eta} \approx 0$ are negligible for $\eta \in \mathcal{E}_{0}$.

In the second term, we claim that all Fourier transforms $\widehat{\mu}_{u}(\xi-\eta) \approx 0$ are negligible for $\xi \in \mathcal{E}_{1}(u), \eta \notin \mathcal{E}_{1}(u) \cup \mathcal{E}_{0}$ : Indeed, denoting by $x=\xi /|\xi|$ and $y=\eta /|\eta|($ note $x \neq y)$, apply Lemma 6.2 with

$$
B=\frac{1}{\lambda} \mathcal{E}_{1}(u) \subset \tau_{u} \operatorname{Cap}\left(w_{0}, \theta_{0}-4 \delta_{0}\right)
$$


Then $y \notin \Omega_{0}$ since $\eta \notin \mathcal{E}_{0}$ hence

$$
\frac{x-y}{|x-y|} \notin \operatorname{Cap}\left(u, 2 \delta_{0}\right)
$$

Since $|\xi|=|\eta|=\lambda$, we have $\frac{\xi-\eta}{|\xi-\eta|}=\frac{x-y}{|x-y|}$. Therefore

$$
\frac{\xi-\eta}{|\xi-\eta|}=\frac{x-y}{|x-y|} \notin \operatorname{Cap}\left(u, 2 \delta_{0}\right)
$$

and hence by the non-stationary phase lemma 6.1, we have

$$
\widehat{\mu}_{u}(\eta-\xi) \ll \frac{1}{|\xi-\eta|^{M}}, \quad \forall M \geq 1
$$

Moreover, since $\xi \in \mathcal{E}_{1}(u)$ and $\eta \notin \mathcal{E}_{1}(u)$,

$$
|\xi-\eta| \geq \operatorname{dist}\left(\mathcal{E}_{1}(u), \mathcal{E} \backslash \mathcal{E}_{1}(u)\right)>\lambda^{\delta(d)}
$$

Hence we get

$$
\widehat{\mu}(\eta-\xi) \ll \frac{1}{\lambda^{N}}, \quad \xi \in \mathcal{E}_{1}(u), \eta \notin \mathcal{E}_{1}(u) \cup \mathcal{E}_{0}
$$

that is the Fourier transforms are negligible as required. Thus the offdiagonal term is negligible, which shows that $\sum_{\xi \in \mathcal{E}_{1}(u)}\left|a_{\xi}\right|^{2}$ is negligible. Since $\frac{1}{\lambda} \mathcal{F}=\Omega_{1}(u) \cap \frac{1}{\lambda} \mathcal{E} \subset \frac{1}{\lambda} \mathcal{E}_{1}(u)$, we get $\left|a_{\xi}\right| \ll \frac{1}{\lambda^{N}}$ if $\xi /|\xi| \in \Omega_{1}(u)$.

Finally, we claim

Proposition 6.4. There is a cap $\Omega_{1}=\operatorname{Cap}\left(w_{1}, \theta_{0}+\delta_{0}\right)$ for which all frequencies $\xi$ in direction $\Omega_{1}$, the Fourier coefficients $a_{\xi}$ are negligible

Proof. We note that the union

$$
\bigcup_{u \in \operatorname{Cap}\left(u_{0}, \delta_{1} / 2\right)} \Omega_{1}(u)=\bigcup_{u \in \operatorname{Cap}\left(u_{0}, \delta_{1} / 2\right)} \operatorname{Cap}\left(\tau_{u} w_{0}, \theta_{0}-5 \delta_{0}\right)
$$

contains a cap $\Omega_{1}=\operatorname{Cap}\left(w_{1}, \theta_{1}\right)$ with $\theta_{1} \geq \theta_{0}+\delta_{0}$.

This follows since the set of reflected centers

$$
\left\{\tau_{u} w_{0}: u \in \operatorname{Cap}\left(u_{0}, \delta_{1} / 2\right)\right\}
$$

contains a cap $\operatorname{Cap}\left(w_{1}, \epsilon_{d}\left(\frac{\delta_{1}}{2}\right)\right)$, where $\epsilon_{d}(\delta)$ is defined in (6.11), since we chose $\delta_{0}$ sufficiently small so that $\epsilon_{d}\left(\frac{\delta_{1}}{2}\right)>6 \delta_{0}$, and hence

$$
\bigcup_{u \in \operatorname{Cap}\left(u_{0}, \delta_{1} / 2\right)} \Omega_{1}(u) \supset \operatorname{Cap}\left(w_{1}, \theta_{0}-5 \delta_{0}+\epsilon_{d}\left(\frac{\delta_{1}}{2}\right)\right) \supset \operatorname{Cap}\left(w_{1}, \theta_{0}+\delta_{0}\right)
$$

Therefore for all frequencies in direction $\Omega_{1}$ the Fourier coefficients $a_{\xi}$ are negligible, since the same holds for each of the small caps $\Omega_{1}(u)$ containing $\Omega_{1}$.

By continuing this process, we see that all coefficients $a_{\xi}$ are negligible, contradicting the normalization $\sum_{\xi}\left|a_{\xi}\right|^{2}=1$. This concludes the proof of Theorem 1.2 . 


\section{Appendix A. The two-Dimensional Case: Using the ABC theorem}

In this section we give a proof of Theorem 1.1 using the function-field "abc theorem" of Brownawell-Masser [3] and Voloch [16]. We recall the statement: Let $K=\mathbb{C}(X)$ be the function field of an algebraic curve of genus $g$ over the complex numbers, $S$ a finite set of places of $K$, and $u_{1}, \ldots u_{m} \in K$ a set of $S$-units, that is rational functions whose zeros and poles lie in $S$. The degree, or height, of a non-constant rational function $x \in K$ is defined as the degree of $K$ over the field extension $\mathbb{C}(x): H(x)=[K: \mathbb{C}(x)]$, which is the number of zeros (or poles) of $x$, counted with multiplicities.

Theorem A.1 ([3], [16]). Let $u_{1}, \ldots, u_{m} \in K$ be non-constant S-units, linearly independent over $\mathbb{C}$, satisfying

$$
\sum_{j=1}^{m} u_{j}=1
$$

Then

$$
\max _{j} H\left(u_{j}\right) \leq \frac{m(m-1)}{2}(2 g-2+\# S)
$$

This result improves that of R.C. Mason [13], where the quadratic term $m(m-1) / 2$ is replaced by a term exponential in $m$, which is not sufficiently strong for our purposes.

A.1. Complexification. Let $\varphi$ be an eigenfunction, $-\Delta \varphi=4 \pi^{2} \lambda^{2} \varphi$, which vanishes on the curve $\Sigma$. Write

$$
\varphi(x)=\sum_{\xi} a_{\xi} e^{2 \pi i\langle\xi, x\rangle}
$$

Let

$$
\operatorname{supp} \widehat{\varphi}=\left\{\xi: a_{\xi} \neq 0\right\}
$$

be the set of frequencies of $\varphi$, and set

$$
r=\# \operatorname{supp} \widehat{\varphi}
$$

to be the number of frequencies; necessarily $r \geq 2$.

We can embed the torus $\mathbb{T}^{2} \simeq S^{1} \times S^{1}$ in $\mathbb{C}^{2}$ via the map $(x, y) \mapsto$ $\left(z_{1}, z_{2}\right)=\left(e^{2 \pi i x}, e^{2 \pi i y}\right)$. This allows us to associate with each trigonometric polynomial (A.3) a Laurent polynomial

$$
F(z)=\sum_{\xi} a_{\xi} z^{\xi}
$$

where for $z=\left(z_{1}, z_{2}\right) \in \mathbb{C}^{2}$ and $\xi=\left(n_{1}, n_{2}\right) \in \mathbb{Z}^{2}$ we denote

$$
z^{\xi}:=z_{1}^{n_{1}} z_{2}^{n_{2}}
$$

We can further write

$$
F\left(z_{1}, z_{2}\right)=\frac{P\left(z_{1}, z_{2}\right)}{z_{1}^{a_{1}} z_{2}^{a_{2}}}
$$


for a unique polynomial $P \in \mathbb{C}\left[z_{1}, z_{2}\right]$ so that $z_{1} \nmid P, z_{2} \nmid P$. If $\varphi \neq a e^{2 \pi i\langle\xi, x\rangle}$ is not composed of a single frequency (which it cannot if we assume that it is real-valued) then $P$ is non-constant. Thus to each trigonometric polynomial $\varphi$ (not a single exponential) we associate the plane curve

$$
X_{P}=\{z: P(z)=0\} \subset \mathbb{C}^{2}
$$

which is possibly reducible and singular.

The nodal set of $\varphi$ must be contained in $X_{P}$, since $z_{i}$ do not vanish on $\mathbb{T}^{2}=S^{1} \times S^{1} \subset \mathbb{C}^{2}$. Thus if $\varphi$ vanishes on the (real) smooth curve $\Sigma \subset \mathbb{T}^{2}$, then $\Sigma$ must be contained in an irreducible component of $X_{P}$ (possibly in more than one component). Thus we get an irreducible component (possibly singular)

$$
X_{D}=\{z: D(z)=0\}
$$

containing $\Sigma$. Here $D \in \mathbb{C}\left[z_{1}, z_{2}\right]$ is an irreducible divisor of $P$ of positive degree. Note that in that case $D\left(z_{1}, z_{2}\right)$ cannot depend only on one of the variables, say on $z_{1}$. Indeed in that case $D\left(z_{1}\right)$ is a one-variable polynomial, and is then irreducible only in the case that it is linear: $D\left(z_{1}\right)=z_{1}-c$, whose zero set is a closed geodesic, contradicting our choice of $\Sigma$.

Let $\lambda_{0}$ be minimal where an eigenfunction $\varphi_{0}$ with eigenvalue $4 \pi^{2} \lambda_{0}^{2}$ vanishes on $\Sigma$. We can choose an irreducible $D_{0} \in \mathbb{C}\left[z_{1}, z_{2}\right]$ so that $\Sigma \subset X_{D_{0}}=$ : $X_{0}$. Let $\mathbb{C}\left(X_{0}\right)$ be the function field of the curve $X_{0}$, that is the field of fractions of the integer domain $\mathbb{C}\left[z_{1}, z_{2}\right] /\left(D_{0}\right)$. The curve $X_{0}$ is irreducible but possibly singular. Let $X \rightarrow X_{0}$ be its normalization, whose ring of regular functions is the integral closure of $\mathbb{C}\left[z_{1}, z_{2}\right] /\left(D_{0}\right)$, and has the same function field as $X_{0}$. The map $X \rightarrow X_{0}$ is one-to-one outside of finitely many points.

Restricting the monomials $z^{\xi}\left(\xi \in \mathbb{Z}^{2}\right)$ to $X_{0}$ gives rational functions which we still denote by $z^{\xi}$, in $\mathbb{C}\left(X_{0}\right)=\mathbb{C}(X)$, which have all their zeros and poles in the set $S_{0}$ given by

$$
S_{0}=X_{0} \cap\left\{\left(z_{1}, z_{2}\right) \in \mathbb{P}^{2}: z_{i}=0, \infty\right\}
$$

Note that $S_{0}$ is finite because $X_{0}$ is not a line of the form $z_{i}=0, \infty$, since $D\left(z_{1}, z_{2}\right)$ depends on both variables. By pulling back to the normalization $X$, we get rational functions, still denoted by $z^{\xi}$, on $X$ which are $S$-units for the pullback $S$ of $S_{0}$ to $X$.

A.2. A lower bound for the height of monomials. In order to apply Theorem A.1, we need to compute the height of the monomials $z^{\xi}$ as rational functions on the curve $X$. The assumption that $\Sigma$ is not a segment of a closed geodesic allows us to obtain a useful lower bound:

Lemma A.2. Suppose that $\Sigma$ is not a segment of a closed geodesic. Then there is some constant $c_{\Sigma}>0$ so that

$$
H\left(z_{1}^{n_{1}} z_{2}^{n_{2}}\right) \geq c_{\Sigma} \max \left(\left|n_{1}\right|,\left|n_{2}\right|\right)
$$

for all $\left(n_{1}, n_{2}\right) \in \mathbb{Z}^{2}$. 
Proof. Let $\operatorname{div} X$ be the vector space of divisors of $X$, that is of (finite) formal sums $\sum_{P \in X} n_{P} P$ (we include points at infinity). The degree of such a divisor is $\sum_{P} n_{p}$. For a rational function $u$ on $X$, we have an associated principal divisor $\operatorname{div} u=\operatorname{div}_{0} u-\operatorname{div}_{\infty} u$ where $\operatorname{div}_{0} u$ and $\operatorname{div}_{\infty} u$ are the divisors of zeros and poles. Then the degree of a principal divisor is zero: $\operatorname{deg} \operatorname{div} u=\operatorname{deg} \operatorname{div}_{0} u-\operatorname{deg} \operatorname{div}_{\infty} u=0$ and the height of $u$ equals

$$
H(u)=\operatorname{deg} \operatorname{div}_{0} u=\operatorname{deg} \operatorname{div}_{\infty} u
$$

On the vector space $\operatorname{div} X$ we have the $\ell^{1}$-norm

$$
\left\|\sum_{P} n_{P} P\right\|_{1}:=\sum_{P}\left|n_{P}\right|
$$

which for a principal divisor equals twice the height of $u$.

$$
\|\operatorname{div} u\|_{1}=\operatorname{deg} \operatorname{div}_{0} u+\operatorname{deg} \operatorname{div}_{\infty} u=2 H(u)
$$

We claim that if $\Sigma$ is not a segment of a closed geodesic, then $\operatorname{div} z_{1}$ and $\operatorname{div} z_{2}$ are linearly independent elements of $\operatorname{div} X$. Indeed, a linear dependence means that there are integers $a_{1}, a_{2} \in \mathbb{Z}$ for which

$$
a_{1} \operatorname{div} z_{1}=a_{2} \operatorname{div} z_{2}
$$

or equivalently that $z_{1}^{a_{1}} / z_{2}^{a_{2}} \equiv c$ is the constant function when restricted to the curve $X_{0}$. That means that on $\Sigma$, we have

$$
e^{2 \pi i\left(a_{1} x_{1}-a_{2} x_{2}\right)}=c
$$

whose zero set is a union of closed geodesics. Hence $\operatorname{div} z_{1}$ and $\operatorname{div} z_{2}$ are linearly independent.

Since $\operatorname{div} z_{1}$ and $\operatorname{div} z_{2}$ are linearly independent, their $\operatorname{span} V$ is a twodimensional vector space in $\operatorname{div} X$. On $V$ we then have two norms: The restriction of the $\ell^{1}$-norm and the $\ell^{\infty}$-norm

$$
\left\|\operatorname{div}\left(z_{1}^{n_{1}} z_{2}^{n_{2}}\right)\right\|_{\infty}=\left\|n_{1} \operatorname{div} z_{1}+n_{2} \operatorname{div} z_{2}\right\|_{\infty}=\max \left(\left|n_{1}\right|,\left|n_{2}\right|\right)
$$

which is indeed a norm since $\operatorname{div} z_{1}$ and $\operatorname{div} z_{2}$ are linearly independent. Since on any finite-dimensional vector space all norms are equivalent, we find that there is some $c=c_{V}>0$ for which

$$
|| \operatorname{div}\left(z_{1}^{n_{1}} z_{2}^{n_{2}}\right)\left\|_{1} \geq c \mid \operatorname{div}\left(z_{1}^{n_{1}} z_{2}^{n_{2}}\right)\right\|_{\infty}=c \max \left(\left|n_{1}\right|,\left|n_{2}\right|\right)
$$

for all $n \in \mathbb{Z}^{2}$, and hence

$$
H\left(z_{1}^{n_{1}} z_{2}^{n_{2}}\right)=\frac{1}{2}\left\|\operatorname{div}\left(z_{1}^{n_{1}} z_{2}^{n_{2}}\right)\right\|_{1} \geq \frac{1}{2} c \max \left(\left|n_{1}\right|,\left|n_{2}\right|\right)
$$

as claimed. 
A.3. Proof of Theorem 1.1. We assume that $\Sigma$ is not a segment of a closed geodesic. We choose $\lambda$ sufficiently large so that

$$
\lambda \gg\left(\# S+2 g_{X}-2\right)^{1+\epsilon}
$$

and show that no eigenfunction $\varphi$ with eigenvalue $4 \pi^{2} \lambda^{2}$ can vanish on $\Sigma$.

Suppose $\lambda$ admits an eigenfunction (A.3) which vanishes on $\Sigma$. Among such eigenfunctions, choose such $\varphi$ with the number of frequencies $r$ being minimal. If $r=2$ then after scaling,

$$
\varphi(x)=e^{2 \pi i\langle\xi, x\rangle}-a e^{2 \pi i\left\langle\xi^{\prime}, x\right\rangle}, \quad a \in \mathbb{C}
$$

and for its nodal set to contain a real point, we need $|a|=1$, that is $a=e^{2 \pi i \alpha}$, $\alpha \in \mathbb{R}$. In that case the nodal set consists of $x \in \mathbb{T}^{2}$ with

$$
\left\langle\xi-\xi^{\prime}, x\right\rangle \in \alpha+\mathbb{Z}
$$

which is a union of straight lines with rational slopes, i.e. closed geodesics. So we may assume $r \geq 3$.

In the expansion (A.3), choose one of the frequencies $\xi_{0}$ and divide all terms in (A.3) by $a_{\xi_{0}} e^{2 \pi i\left\langle\xi_{0}, x\right\rangle}$ to get a relation:

$$
\sum_{\xi_{0} \neq \xi \in \operatorname{supp} \widehat{\varphi}}-\frac{a_{\xi}}{a_{\xi_{0}}} z^{\xi-\xi_{0}}=1
$$

Set

$$
u_{\xi}:=-\frac{a_{\xi}}{a_{\xi_{0}}} z^{\xi-\xi_{0}} \in \mathbb{C}\left(X_{0}\right)
$$

Then we get a relation in $\mathbb{C}\left(X_{0}\right)=\mathbb{C}(X)$ (an $S$-unit equation)

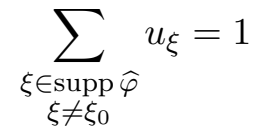

where $u_{\xi}$ are linearly independent, by the minimality assumption on $\varphi$. To the relation (A.12) we apply the "abc-Theorem" (Theorem A.1) which says that if $r \geq 3$ then

$$
\max \left(H\left(u_{\xi}\right): \xi_{0} \neq \xi \in \operatorname{supp} \widehat{\varphi}\right) \leq \frac{(r-1)(r-2)}{2}\left(\# S+2 g_{X}-2\right)
$$

where $g_{X}$ is the genus of the smooth curve $X$. Since

$$
H\left(u_{\xi}\right)=H\left(z^{\xi-\xi_{0}}\right) \geq c_{\Sigma}\left\|\xi-\xi_{0}\right\|_{\infty}
$$

by Lemma A.2, we find that

$$
\max _{\xi \neq \xi_{0}}\left\|\xi-\xi_{0}\right\|_{\infty} \ll \frac{(r-1)(r-2)}{2}\left(\# S+2 g_{X}-2\right)
$$

Now the number of frequencies $r$ is at most the total number of lattice points on the circle $|x|=\lambda$, hence is bounded by $r \ll \lambda^{\epsilon}$ for all $\epsilon>0$. Thus by (A.14) we find that all frequencies of $\varphi$ are contained in a box of size $\ll \lambda^{\epsilon}$ around $\xi_{0}$. By Jarnik's theorem (Theorem 2.1), any arc of size $\ll \lambda^{1 / 3}$ 
contains at most two lattice points, hence this forces $r=2$ contradicting our assumption $r \geq 3$. This gives a contradiction for $\lambda$ sufficiently large.

\section{REFERENCES}

[1] J. Bourgain and Z. Rudnick, Restriction of toral eigenfunctions to hypersurfaces. C. R. Math. Acad. Sci. Paris 347 (2009), no. 21-22, 1249-1253.

[2] J. Bourgain and Z. Rudnick, in preparation.

[3] W. D. Brownawell and D.W. Masser, Vanishing sums in function fields. Math. Proc. Cambridge Philos. Soc. 100 (1986), no. 3, 427-434.

[4] N. Burq, P. Gérard, and N. Tzvetkov. Restrictions of the Laplace-Beltrami eigenfunctions to submanifolds. Duke Math. J. 138 (2007), no. 3, 445-486.

[5] H. Donnelly and C. Fefferman, Nodal sets of eigenfunctions on Riemannian manifolds. Invent. Math. 93 (1988), no. 1, 161-183.

[6] C. S. Herz Fourier transforms related to convex sets. Ann. of Math. (2) 75 (1962), 81-92.

[7] E. Hlawka, Über Integrale auf konvexen Körpern. I. Monatsh. Math. 54, (1950). 1-36.

[8] JB Holt, The irreducibility of Legendre's polynomials. Proceedings of the London Mathematical Society s2-11 (1913), 351-356.

[9] R. Hu, $L^{p}$ norm estimates of eigenfunctions restricted to submanifolds, Forum Mathematicum 21, No. 6 (2009), 1021-1052.

[10] Hildegard Ille, Jbuch. der Dissertationen, Berlin, 1924.

[11] V. Jarnik. Über die Gitterpunkte auf konvexen Kurven. Math. Z. 24 (1926), no. 1, $500-518$.

[12] W. Littman, Fourier transforms of surface-carried measures and differentiability of surface averages. Bull. Amer. Math. Soc. 691963 766-770.

[13] R.C. Mason, Norm form equations. I. J. Number Theory 22 (1986), no. 2, 190-207.

[14] P. Sarnak, Letter to Reznikov, June 2008, see www.math.princeton.edu/sarnak/

[15] T.J. Stieltjes, Letter No. 275 of Oct. 2, 1890, in Correspondance d'Hermite et de Stieltjes, vol 2, Gauthier-Villars, Paris, 1905.

[16] J.F. Voloch, Diagonal equations over function fields. Bol. Soc. Brasil. Mat. 16 (1985), no. 2, 2939.

[17] J.H. Wahab, New cases of irreducibility for Legendre polynomials. Duke Math. J. 19, (1952). 165-176.

[18] J.H. Wahab, New cases of irreducibility for Legendre polynomials. II. Duke Math. J. 271960 481-482.

School of Mathematics, Institute for Advanced Study, Princeton, NJ 08540

E-mail address: bourgain@ias.edu

Raymond and Beverly Sackler School of Mathematical Sciences, Tel Aviv University, Tel Aviv 69978, IsRael

E-mail address: rudnick@post.tau.ac.il 